\title{
Additive problems with prime numbers of special type
}

\author{
by \\ D. I. TOlev (Plovdiv)
}

1. Introduction and statement of the results. In 1937 I. M. Vinogradov [28] proved that for every sufficiently large odd integer $n$ the equation

$$
p_{1}+p_{2}+p_{3}=n
$$

has a solution in prime numbers. It is still not known whether every sufficiently large even integer $n$ can be represented as

$$
p_{1}+p_{2}=n,
$$

where $p_{1}, p_{2}$ are primes. Denote by $E(N)$ the number of even integers not exceeding $N$ and not representable in the form (1.2). Many researchers have worked to obtain non-trivial upper bounds for this quantity. The most important result belongs to Montgomery and Vaughan [19]. They proved in 1975 that there exists an effective constant $\delta>0$ such that $E(N) \ll N^{1-\delta}$.

Another important approach for studying the equation (1.2) is by the use of sieve methods. The strongest result in this direction belongs to Chen [3]. Denote, as usual, by $\mathrm{P}_{r}$ any integer with no more than $r$ prime factors, counted according to multiplicity. In 1973 Chen proved that every sufficiently large even $n$ can be represented as a sum of a prime and a $\mathrm{P}_{2}$. He also proved that there are infinitely many primes $p$ such that $p+2=\mathrm{P}_{2}$.

In 1938 Hua studied the equation

$$
p_{1}^{2}+p_{2}^{2}+p_{3}^{2}=n
$$

for solvability in prime numbers. By elementary considerations one may see that necessary conditions for the solvability of $(1.3)$ are $n \equiv 3(\bmod 24)$ and $n \not \equiv 0(\bmod 5)$. Denote by $E_{1}(N)$ the number of integers $n \leq N$ satisfying these congruences and which are not representable in the form (1.3). Hua [8] proved the existence of a constant $B>0$ such that $E_{1}(N) \ll N(\log N)^{-B}$. Schwarz [22] proved this estimate with arbitrarily large $B>0$. In 1993

2000 Mathematics Subject Classification: 11P32, 11N36. 
M.-C. Leung and M.-C. Liu [14] showed that $E_{1}(N) \ll N^{1-\delta}$ for some $\delta>0$. Short-interval versions of this problem were considered by J. Liu and T. Zhan [15] and Mikawa [16].

As a corollary to his theorem Hua established that the equation

$$
p_{1}^{2}+p_{2}^{2}+p_{3}^{2}+p_{4}^{2}+p_{5}^{2}=n
$$

is solvable in primes provided that $n$ is sufficiently large and satisfies $n \equiv 5$ $(\bmod 24)$.

In 1939 van der Corput [26] established that there exist infinitely many arithmetic progressions of three different primes. The corresponding question for progressions of four or more primes is still open. In 1981, however, Heath-Brown [6] proved that there exist infinitely many arithmetic progressions of four different terms, three of which are primes and the fourth is $\mathrm{P}_{2}$.

The work of Heath-Brown motivated the author to study additive problems with primes $p$ such that $p+2$ is almost-prime. In [21] Peneva and the author proved that there exist infinitely many arithmetic progressions of three different primes $p_{1}, p_{2}, p_{3}$ such that $\left(p_{1}+2\right)\left(p_{2}+2\right)=\mathrm{P}_{9}$. Later the author used some ideas of Brüdern and Fouvry [1] and Heath-Brown and was able to impose a multiplicative restriction on $p_{3}+2$ as well. It was proved in [23] that there exist infinitely many arithmetic progressions of three different primes $p_{1}, p_{2}, p_{3}=\frac{1}{2}\left(p_{1}+p_{2}\right)$ such that $p_{1}+2=\mathrm{P}_{5}$, $p_{2}+2=\mathrm{P}_{5}^{\prime}, p_{3}+2=\mathrm{P}_{8}$. Peneva [20] used the method of [23] to consider the corresponding problem for the equation (1.1).

Recently the author considered the equation (1.4) for solvability in primes of the type described above. It was established in [24] that if $n$ is a sufficiently large integer satisfying $n \equiv 5(\bmod 24)$ then $(1.4)$ has a solution in primes $p_{1}, \ldots, p_{5}$ such that each of the numbers $p_{1}+2, p_{2}+2, p_{3}+2$, $p_{4}+2$ is $\mathrm{P}_{6}$ and $p_{5}+2=\mathrm{P}_{7}$. We should also mention the earlier result [13] of Laporta and the author, which is somewhat related to [24].

In the present paper we study the equations (1.2) and (1.3) with variables prime numbers of the type mentioned above. We prove that they are solvable for almost all $n$ satisfying some natural congruence conditions. The following theorems hold:

Theorem 1. Denote by $\mathcal{K}$ the set of integers $n$ for which the equation (1.3) has a solution in primes $p_{1}, p_{2}, p_{3}$ such that $p_{1}+2=\mathrm{P}_{5}, p_{2}+2=\mathrm{P}_{5}^{\prime}$, $p_{3}+2=\mathrm{P}_{8}$. Consider the set

$$
\mathcal{F}=\{n \leq N: n \equiv 3(\bmod 24), n \not \equiv 0(\bmod 5)\} \backslash \mathcal{K}
$$

and let $\mathcal{Y}(N)$ be its cardinality. Then for arbitrarily large $B>0$ we have

$$
\mathcal{Y}(N) \ll N(\log N)^{-B}
$$


TheORem 2. Denote by $\mathcal{K}_{0}$ the set of integers $n$ for which the equation (1.2) has a solution in different primes $p_{1}, p_{2}$ such that $p_{1}+2=\mathrm{P}_{5}, p_{2}+2$ $=\mathrm{P}_{7}$. Consider the set

$$
\mathcal{F}_{0}=\{n \leq N: n \equiv 4(\bmod 6)\} \backslash \mathcal{K}_{0}
$$

and let $\mathcal{Y}_{0}(N)$ be its cardinality. Then for arbitrarily large $B>0$ we have

$$
\mathcal{Y}_{0}(N) \ll N(\log N)^{-B} .
$$

From Theorem 1 we easily obtain

COROllary 1. For every sufficiently large integer $n \equiv 5(\bmod 24)$ the equation (1.4) has a solution in prime numbers $p_{1}, \ldots, p_{5}$ such that $p_{1}+2$ $=\mathrm{P}_{2}, p_{2}+2=\mathrm{P}_{2}^{\prime}, p_{3}+2=\mathrm{P}_{5}, p_{4}+2=\mathrm{P}_{5}^{\prime}, p_{5}+2=\mathrm{P}_{8}$.

Pr o of. Consider the sets of primes

$$
\mathfrak{A}=\left\{p \leq \frac{1}{2} \sqrt{n}: p \equiv 11(\bmod 30), p+2=\mathrm{P}_{2}\right\}
$$

and

$$
\mathfrak{A}^{\prime}=\left\{p \leq \frac{1}{2} \sqrt{n}: p \equiv 17(\bmod 30), p+2=\mathrm{P}_{2}\right\} .
$$

Applying the arguments of Chen we establish that the cardinalities of $\mathfrak{A}$ and $\mathfrak{A}^{\prime}$ are $\gg \sqrt{n}(\log n)^{-2}$.

Suppose that $n \not \equiv 2(\bmod 5)$. Consider the set $\left\{n-p^{2}-q^{2}: p, q \in \mathfrak{A}\right\}$. It is not difficult to see that it contains $\gg n(\log n)^{-9}$ distinct integers $k$ satisfying $k \equiv 3(\bmod 24), k \neq \equiv 0(\bmod 5)$. It remains to apply Theorem 1 .

If $n \equiv 2(\bmod 5)$ then we consider the set $\left\{n-p^{2}-q^{2}: p \in \mathfrak{A}, q \in \mathfrak{A}^{\prime}\right\}$ and then we proceed as in the first case.

Similarly, from Theorem 2 we obtain the following corollaries:

COROllary 2. For every sufficiently large integer $n \equiv 3(\bmod 6)$ the equation (1.1) has a solution in prime numbers $p_{1}, p_{2}, p_{3}$ such that $p_{1}+2=$ $\mathrm{P}_{2}, p_{2}+2=\mathrm{P}_{5}, p_{3}+2=\mathrm{P}_{7}$.

COROLLARY 3. There are infinitely many arithmetic progressions of three different primes $p_{1}, p_{2}, p_{3}=\frac{1}{2}\left(p_{1}+p_{2}\right)$ such that $p_{3}+2=\mathrm{P}_{2}, p_{1}+2=\mathrm{P}_{5}$, $p_{2}+2=\mathrm{P}_{7}$.

To prove the theorems we apply the method of [20], [23] and [24]. In many places we omit the calculations because they are similar to those in the papers mentioned above. We present only the proof of Theorem 1 . The proof of Theorem 2 is simpler and it was briefly explained in [25].

In Section 2 we introduce the notations and state a Proposition, which is of some independent interest. It asserts that the expected asymptotic formula for the number of the solutions of (1.3) in primes from arithmetic progressions is valid "on average". 
In Section 3 we prove Theorem 1 . We consider the sum $\Gamma$ defined by (3.3) and we show that it is not large. On the other hand, we estimate it from below using the vector sieve of Iwaniec [10] and Brüdern-Fouvry [1]. We find that if the cardinality $\mathcal{Y}(N)$ of the set $\mathcal{F}$ were large then the lower bound for $\Gamma$ would be considerably larger than $\Gamma$, which is not possible. This proves the theorem.

In Sections 4 and 5 we prove the Proposition by means of the circle method. We consider the minor arcs in Section 4. The crucial point is formula (4.4) which gives a non-trivial estimate for a double exponential sum. The idea is due to Heath-Brown, who pointed out to the author that non-trivial estimates exist for such kind of sums. We also find an estimate for the mean value of the same sum.

To treat the major arcs we work as in [13], [21], [23], [24]. We find asymptotic formulae for exponential sums over primes lying in arithmetic progressions. It appears that the error terms of these formulae are small "on average" and applying the Bombieri-Vinogradov theorem we find that their contribution is negligible. The computations are presented in Section 5 .

Acknowledgements. The main part of this research was done during the author's visit to the Institute of Mathematics of the University of Oxford. The author thanks the Royal Society for financial support, the staff of the Institute for the excellent working conditions and also Plovdiv University Scientific Fund (grant PU2-MM) for covering some other expenses.

The author is especially grateful to Professor D. R. Heath-Brown for useful and interesting discussions and valuable remarks.

2. Notations and statement of the Proposition. The letter $p$ is reserved for prime numbers. Lower case Latin letters (except $x, y, z$ and $p$ ) denote integers. Other letters denote real or complex numbers and the meaning is always clear from the context. As usual, $\mu(n), \varphi(n), \Lambda(n), \nu(n)$ denote the Möbius function, Euler's function, von Mangoldt's function and the number of distinct prime factors of $n$, respectively; $\tau_{k}(n)$ denotes the number of solutions of the equation $m_{1} \ldots m_{k}=n$ in integers $m_{1}, \ldots, m_{k} ; \tau(n)=\tau_{2}(n)$. We denote by $\left(m_{1}, \ldots, m_{k}\right)$ and $\left[m_{1}, \ldots, m_{k}\right]$ the greatest common divisor and least common multiple of $m_{1}, \ldots, m_{k}$, respectively. For real $y, z$, however, $(y, z)$ denotes the open interval on the real line with endpoints $y$ and $z$. Instead of $m \equiv n(\bmod k)$ we sometimes write for simplicity $m \equiv n(k)$. As usual $\|y\|$ denotes the distance from $y$ to the nearest integer and $e(y)=$ $\exp (2 \pi i y)$. We write $p^{l} \| n$ if $p^{l} \mid n$ and $p^{l+1} \nmid n$. The Legendre symbol is denoted by $(\dot{\bar{p}})$. For positive $U$ and $V$ we write $U \asymp V$ instead of $U \ll V \ll U$.

Suppose that $A \geq 10000$ is a constant. If not explicitly specified, constants in $\mathcal{O}$-terms and Vinogradov's symbols are absolute or depend only on $A$. Let $N$ be sufficiently large and put $X=\sqrt{N}$ and $\mathcal{L}=\log X$. 
A central point in our paper is the study of the sum

$$
I\left(n ; k_{1}, k_{2}, k_{3}\right)=\sum_{\substack{p_{1}^{2}+p_{2}^{2}+p_{3}^{2}=n \\ p_{i}+2 \equiv 0\left(\bmod k_{i}\right) \\ i=1,2,3}} \log p_{1} \log p_{2} \log p_{3},
$$

where $k_{1}, k_{2}, k_{3}$ are odd squarefree numbers and $n \leq N$. It is clear that

$$
I\left(n ; k_{1}, k_{2}, k_{3}\right)=\int_{0}^{1} S_{k_{1}}(\alpha) S_{k_{2}}(\alpha) S_{k_{3}}(\alpha) e(-n \alpha) d \alpha,
$$

where

$$
S_{k}(\alpha)=\sum_{\substack{p \leq X \\ p+2 \equiv 0(k)}} \log p e\left(\alpha p^{2}\right) .
$$

Define

$$
\begin{gathered}
Q=\mathcal{L}^{1000 A}, \quad \tau=X^{2} \mathcal{L}^{-2000 A} \\
E_{1}=\bigcup_{q<Q} \bigcup_{\substack{a=0 \\
(a, q)=1}}^{q-1}\left(\frac{a}{q}-\frac{1}{q \tau}, \frac{a}{q}+\frac{1}{q \tau}\right), \quad E_{2}=\left(-\frac{1}{\tau}, 1-\frac{1}{\tau}\right) \backslash E_{1} .
\end{gathered}
$$

We have

$$
I\left(n ; k_{1}, k_{2}, k_{3}\right)=I_{1}+I_{2},
$$

where

$$
I_{j}=\int_{E_{j}} S_{k_{1}}(\alpha) S_{k_{2}}(\alpha) S_{k_{3}}(\alpha) e(-n \alpha) d \alpha, \quad j=1,2 .
$$

Define

$$
\begin{gathered}
s_{k}(a, q)=\frac{\varphi((k, q))}{\varphi(q)} \sum_{\substack{1 \leq m \leq q \\
(m, q)=1 \\
m+2 \equiv 0((k, q))}} e\left(\frac{a m^{2}}{q}\right), \\
t(q)=t\left(q ; n ; k_{1}, k_{2}, k_{3}\right) \quad \sum_{\substack{0 \leq a \leq q-1 \\
(a, q)=1}} s_{k_{1}}(a, q) s_{k_{2}}(a, q) s_{k_{3}}(a, q) e\left(-n \frac{a}{q}\right) .
\end{gathered}
$$

The function $t(q)$ is multiplicative with respect to $q$. Using the definition (2.7) of $s_{k}(a, q)$ and the properties of the Gauss sum (see, for example, Hua [9], Chapter 7) it is not difficult to compute $t\left(p^{l}\right)$.

We find that if $n \equiv 3(\bmod 8)$ and $k_{1}, k_{2}, k_{3}$ are odd integers then

$$
t(2)=1, \quad t(4)=2, \quad t(8)=4, \quad t\left(2^{l}\right)=0 \quad \text { for } l>3 .
$$


Define

$$
\begin{aligned}
& h_{0}(p)= \begin{cases}\frac{\left(\frac{-n}{p}\right) p^{2}+\left(3\left(\frac{n}{p}\right)+3\left(\frac{-1}{p}\right)\right) p+1}{(p-1)^{3}} & \text { if } p \nmid n, \\
\frac{-3\left(\frac{-1}{p}\right) p-1}{(p-1)^{2}} & \text { if } p \mid n,\end{cases} \\
& h_{1}(p)= \begin{cases}\frac{\left(-2\left(\frac{n-4}{p}\right)-\left(\frac{-1}{p}\right)\right) p-1}{(p-1)^{2}} & \text { if } p \nmid n-4, \\
\frac{\left(\frac{-1}{p}\right) p+1}{p-1} & \text { if } p \mid n-4,\end{cases} \\
& h_{2}(p)= \begin{cases}\frac{\left(\frac{n-8}{p}\right) p+1}{p-1} & \text { if } p \nmid n-8, \\
-1 & \text { if } p \mid n-8,\end{cases} \\
& h_{3}(p)= \begin{cases}-1 & \text { if } p \nmid n-12, \\
p-1 \quad \text { if } p \mid n-12 .\end{cases}
\end{aligned}
$$

If $p>2$ and $k_{1}, k_{2}, k_{3}$ are squarefree integers then we have

$$
\begin{aligned}
& t(p)= \begin{cases}h_{0}(p) & \text { if } p \nmid k_{1} k_{2} k_{3}, \\
h_{1}(p) & \text { if } p \| k_{1} k_{2} k_{3}, \\
h_{2}(p) & \text { if } p^{2} \| k_{1} k_{2} k_{3}, \\
h_{3}(p) & \text { if } p^{3} \| k_{1} k_{2} k_{3},\end{cases} \\
& t\left(p^{l}\right)=0 \quad \text { if } l>1 .
\end{aligned}
$$

We leave the calculations to the reader.

Define

$$
\mathfrak{S}=\mathfrak{S}\left(n ; Q ; k_{1}, k_{2}, k_{3}\right)=8 \prod_{2<p<Q}\left(1+t\left(p ; n ; k_{1}, k_{2}, k_{3}\right)\right)
$$

We write

$$
I\left(n ; k_{1}, k_{2}, k_{3}\right)=\frac{\pi}{4} \sqrt{n} \frac{\mathfrak{S}\left(n ; Q ; k_{1}, k_{2}, k_{3}\right)}{\varphi\left(k_{1}\right) \varphi\left(k_{2}\right) \varphi\left(k_{3}\right)}+\mathfrak{R}\left(n ; Q ; k_{1}, k_{2}, k_{3}\right) .
$$

The first summand arises from the application of the circle method. We cannot find a non-trivial estimate for the remainder $\mathfrak{R}$ for individual $n, k_{1}$, $k_{2}, k_{3}$, but we prove that it is small on average. We have:

Proposition. Suppose that

$$
K_{1}, K_{2} \leq X^{1 / 2} \mathcal{L}^{-20000 A}, \quad K_{3} \leq X^{1 / 3} \mathcal{L}^{-20000 A}
$$

and let $\beta_{i}\left(k_{i}\right), k_{i} \leq K_{i}, i=1,2,3$, be complex numbers satisfying

$$
\beta_{i}(k)=0 \quad \text { if } 2 \mid k \text { or } \mu(k)=0 ; \quad\left|\beta_{i}(k)\right| \leq \tau(k) .
$$


Then for

$$
\mathcal{U}=\sum_{\substack{n \leq N \\ n \equiv 3(24) \\ n \neq 0(5)}}\left|\sum_{\substack{k_{i} \leq K_{i} \\ i=1,2,3}} \beta_{1}\left(k_{1}\right) \beta_{2}\left(k_{2}\right) \beta_{3}\left(k_{3}\right) \mathfrak{R}\left(n ; Q ; k_{1}, k_{2}, k_{3}\right)\right|
$$

we have

$$
\mathcal{U} \ll X^{3} \mathcal{L}^{-A} .
$$

For brevity we will write $\sum_{n \leq N}^{*}$ to emphasize that the summation is taken over the integers $n$ satisfying $n \equiv 3(\bmod 24)$ and $n \not \equiv 0(\bmod 5)$.

To prove the Proposition we consider

$$
\begin{aligned}
& \mathcal{U}_{1}=\sum_{n \leq N}^{*} \mid \sum_{\substack{k_{i} \leq K_{i} \\
i=1,2,3}} \beta_{1}\left(k_{1}\right) \beta_{2}\left(k_{2}\right) \beta_{3}\left(k_{3}\right) \\
& \times\left(I_{1}-\frac{\pi}{4} \sqrt{n} \frac{\mathfrak{S}\left(n ; Q ; k_{1}, k_{2}, k_{3}\right)}{\varphi\left(k_{1}\right) \varphi\left(k_{2}\right) \varphi\left(k_{3}\right)}\right) \mid, \\
& \mathcal{U}_{2}=\sum_{n \leq N}\left|\sum_{\substack{k_{i} \leq K_{i} \\
i=1,2,3}} \beta_{1}\left(k_{1}\right) \beta_{2}\left(k_{2}\right) \beta_{3}\left(k_{3}\right) I_{2}\right| .
\end{aligned}
$$

Obviously

$$
\mathcal{U} \ll \mathcal{U}_{1}+\mathcal{U}_{2} .
$$

We study $\mathcal{U}_{2}$ in Section 4 and $\mathcal{U}_{1}$ in Section 5 and we prove that

$$
\mathcal{U}_{1}, \mathcal{U}_{2} \ll X^{3} \mathcal{L}^{-A} \text {. }
$$

The estimate (2.19) is a consequence of (2.22) and (2.23).

Note that only in the proof of the inequality (4.4) do we need the tight restriction on $K_{3}$ imposed by (2.17). So the validity of (4.4) for larger values of $K_{3}$ would certainly imply an improvement of Theorem 1.

3. Proof of Theorem 1. Let $\mathcal{F}$ be the set defined in Theorem 1 . We put

$$
Q_{0}=\mathcal{L}^{0.6}, \quad z_{1}=z_{2}=X^{0.167}, \quad z_{3}=X^{0.116} .
$$

Let $\mathfrak{R}=\{p \geq 11: p \nmid n-4\} \cup\{p \geq 11: p \mid n-4, p \equiv 1(\bmod 4)\}$. We define

$$
\mathcal{B}_{0}=\prod_{3 \leq p<Q_{0}} p, \quad \mathcal{P}_{0}=\prod_{\substack{Q_{0} \leq p<Q \\ p \in \mathfrak{R}}} p, \quad \mathcal{P}_{i}=\prod_{Q \leq p<z_{i}} p, \quad i=1,2,3 .
$$


Consider the sum

$$
\Gamma=\sum_{n \in \mathcal{F}} \sum_{\begin{array}{c}
p_{1}^{2}+p_{2}^{2}+p_{3}^{2}=n \\
\left(p_{i}+2, \mathcal{B}_{0} \mathcal{P}_{0} \mathcal{P}_{i}\right)=1 \\
i=1,2,3
\end{array}} \log p_{1} \log p_{2} \log p_{3}=\sum_{n \in \mathcal{F}} w(n),
$$

say. Suppose that $w(n)>0$ for some $n \in \mathcal{F}$. Then there exist primes $p_{1}, p_{2}, p_{3}$ satisfying the conditions imposed in the inner sum of formula (3.3). For one of them, $p_{1}$ say, we should have $\left(p_{1}+2, \prod_{p<z_{1}} p\right)>1$, otherwise we would have $\left(p_{i}+2, \prod_{p<z_{i}} p\right)=1$ for $i=1,2,3$, which would contradict the definitions of $\mathcal{F}$ and $z_{i}$.

If $p_{1}=2$ then $w(n) \ll \mathcal{L}^{3} \sum_{n=m_{1}^{2}+m_{2}^{2}+4} 1$.

If $p_{1}>2$ then $p_{1}+2$ would have a prime factor $p>2$ such that $p \mid n-4$ and $p \equiv 3(\bmod 4)$. Hence $p_{2}^{2}+p_{3}^{2} \equiv 0(\bmod p)$, which implies $p_{2}=p_{3}=p$ and, therefore $w(n) \ll \mathcal{L}^{3} \sum_{p \mid n-4} 1$.

Consequently,

$$
\Gamma \ll \mathcal{L}^{3}\left(\sum_{m_{1}^{2}+m_{2}^{2}+4 \leq N} 1+\sum_{n \leq N} \tau(n-4)\right) \ll X^{2} \mathcal{L}^{4} .
$$

Now we will use the vector sieve to estimate $\Gamma$ from below. First we get rid of the summands corresponding to integers $n$ such that $n-4$ has many distinct prime factors. From this point onwards $\sum^{\#}$ stands for a sum over $n$ such that $\nu(n-4) \leq A \log \mathcal{L}$. For technical reasons we sieve separately by the primes from the intervals $\left[3, Q_{0}\right),\left[Q_{0}, Q\right)$ and $[Q, \infty)$. From the basic property of Möbius' function we get

$$
\Gamma \geq \sum_{n \in \mathcal{F}}^{\#} \sum_{p_{1}^{2}+p_{2}^{2}+p_{3}^{2}=n} \log p_{1} \log p_{2} \log p_{3} \Phi_{1} \Phi_{2} \Phi_{3} \Lambda_{1} \Lambda_{2} \Lambda_{3} \Lambda_{4} \Lambda_{5} \Lambda_{6},
$$

where

$$
\begin{aligned}
\Phi_{i} & =\sum_{d \mid\left(p_{i}+2, \mathcal{B}_{0}\right)} \mu(d), \quad i=1,2,3 ; \\
\Lambda_{i} & = \begin{cases}\sum_{d \mid\left(p_{i}+2, \mathcal{P}_{i}\right)} \mu(d) & \text { for } i=1,2,3, \\
\sum_{d \mid\left(p_{i-3}+2, \mathcal{P}_{0}\right)} \mu(d) & \text { for } i=4,5,6 .\end{cases}
\end{aligned}
$$

Define

$$
\begin{aligned}
& D_{1}=D_{2}=X^{1 / 2} \exp \left(-4 \mathcal{L}^{0.6}\right), \\
& D_{3}=X^{1 / 3} \exp \left(-4 \mathcal{L}^{0.6}\right), \quad D_{0}=\exp \left(\mathcal{L}^{0.6}\right) .
\end{aligned}
$$

By $\lambda_{i}^{ \pm}(d)$ we denote Rosser's weights of order $D_{i}, 0 \leq i \leq 3$ (see Iwaniec [11], [12] for the definition). In particular, we have

$$
\left|\lambda_{i}^{ \pm}(d)\right| \leq 1, \quad \lambda_{i}^{ \pm}(d)=0 \quad \text { for } d \geq D_{i}, \quad 0 \leq i \leq 3
$$


Denote

$$
\Lambda_{i}^{ \pm}= \begin{cases}\sum_{d \mid\left(p_{i}+2, \mathcal{P}_{i}\right)} \lambda_{i}^{ \pm}(d) & \text { for } i=1,2,3, \\ \sum_{d \mid\left(p_{i-3}+2, \mathcal{P}_{0}\right)} \lambda_{i}^{ \pm}(d) & \text { for } i=4,5,6 .\end{cases}
$$

By the properties of Rosser's weights (see Iwaniec [11], [12]) we have $\Lambda_{i}^{-} \leq \Lambda_{i} \leq \Lambda_{i}^{+}, 1 \leq i \leq 6$. We apply the inequality

$$
\begin{aligned}
\Lambda_{1} \Lambda_{2} \Lambda_{3} \Lambda_{4} \Lambda_{5} \Lambda_{6} \geq & \Lambda_{1}^{-} \Lambda_{2}^{+} \Lambda_{3}^{+} \Lambda_{4}^{+} \Lambda_{5}^{+} \Lambda_{6}^{+}+\Lambda_{1}^{+} \Lambda_{2}^{-} \Lambda_{3}^{+} \Lambda_{4}^{+} \Lambda_{5}^{+} \Lambda_{6}^{+} \\
& +\Lambda_{1}^{+} \Lambda_{2}^{+} \Lambda_{3}^{-} \Lambda_{4}^{+} \Lambda_{5}^{+} \Lambda_{6}^{+}+\Lambda_{1}^{+} \Lambda_{2}^{+} \Lambda_{3}^{+} \Lambda_{4}^{-} \Lambda_{5}^{+} \Lambda_{6}^{+} \\
& +\Lambda_{1}^{+} \Lambda_{2}^{+} \Lambda_{3}^{+} \Lambda_{4}^{+} \Lambda_{5}^{-} \Lambda_{6}^{+}+\Lambda_{1}^{+} \Lambda_{2}^{+} \Lambda_{3}^{+} \Lambda_{4}^{+} \Lambda_{5}^{+} \Lambda_{6}^{-} \\
& -5 \Lambda_{1}^{+} \Lambda_{2}^{+} \Lambda_{3}^{+} \Lambda_{4}^{+} \Lambda_{5}^{+} \Lambda_{6}^{+} .
\end{aligned}
$$

The proof is the same as in Lemma 13 of [1]. Using this inequality and (3.5) we get

$$
\Gamma \geq \sum_{i=1}^{6} \Gamma_{i}-5 \Gamma_{7}
$$

where

$$
\Gamma_{1}=\sum_{n \in \mathcal{F}}^{\#} \sum_{p_{1}^{2}+p_{2}^{2}+p_{3}^{2}=n} \log p_{1} \log p_{2} \log p_{3} \Phi_{1} \Phi_{2} \Phi_{3} \Lambda_{1}^{-} \Lambda_{2}^{+} \Lambda_{3}^{+} \Lambda_{4}^{+} \Lambda_{5}^{+} \Lambda_{6}^{+} .
$$

The definition of the other sums $\Gamma_{i}$ is clear. We change the order of summation to get

$$
\begin{aligned}
\Gamma_{1}= & \sum_{n \in \mathcal{F}}^{\#} \sum_{\begin{array}{c}
\nu_{i}\left|\mathcal{B}_{0}, \delta_{i}\right| \mathcal{P}_{0} \\
d_{i} \mid \mathcal{P}_{i}, i=1,2,3
\end{array}} \mu\left(\nu_{1}\right) \mu\left(\nu_{2}\right) \mu\left(\nu_{3}\right) \lambda_{1}^{-}\left(d_{1}\right) \lambda_{2}^{+}\left(d_{2}\right) \lambda_{3}^{+}\left(d_{3}\right) \\
& \times \lambda_{0}^{+}\left(\delta_{1}\right) \lambda_{0}^{+}\left(\delta_{2}\right) \lambda_{0}^{+}\left(\delta_{3}\right) I\left(n ; \nu_{1} \delta_{1} d_{1}, \nu_{2} \delta_{2} d_{2}, \nu_{3} \delta_{3} d_{3}\right),
\end{aligned}
$$

where $I\left(n ; k_{1}, k_{2}, k_{3}\right)$ is defined by $(2.1)$.

Using formula $(2.16)$ we split $\Gamma_{1}$ into two parts:

$$
\Gamma_{1}=\Gamma_{1}^{\prime}+\Gamma_{1}^{\prime \prime},
$$

where $\Gamma_{1}^{\prime}$ and $\Gamma_{1}^{\prime \prime}$ are the contributions from the main term and error term of the formula (2.16) respectively.

Consider $\Gamma_{1}^{\prime \prime}$. We write it in the form

$$
\Gamma_{1}^{\prime \prime}=\sum_{n \in \mathcal{F}}^{\#} \sum_{\substack{k_{i} \leq \mathcal{B}_{0} D_{0} D_{i} \\ i=1,2,3}} \gamma_{1}\left(k_{1}\right) \gamma_{2}\left(k_{2}\right) \gamma_{3}\left(k_{3}\right) \Re\left(n ; Q ; k_{1}, k_{2}, k_{3}\right),
$$

where

$$
\gamma_{1}(k)=\sum_{\substack{\nu\left|\mathcal{B}_{0}, \delta\right| \mathcal{P}_{0}, d \mid \mathcal{P}_{1} \\ \nu \delta d=k}} \mu(\nu) \lambda_{0}^{+}(\delta) \lambda_{1}^{-}(d)
$$




$$
\gamma_{i}(k)=\sum_{\substack{\nu\left|\mathcal{B}_{0}, \delta\right| \mathcal{P}_{0}, d \mid \mathcal{P}_{i} \\ \nu \delta d=k}} \mu(\nu) \lambda_{0}^{+}(\delta) \lambda_{i}^{+}(d) \quad \text { for } i=2,3 .
$$

Now we use (3.1), (3.2), (3.7), (3.8) and apply the Proposition to find that

$$
\Gamma_{1}^{\prime \prime} \ll X^{3} \mathcal{L}^{-A} \text {. }
$$

Consider $\Gamma_{1}^{\prime}$. Using the definitions (2.8) and (2.15) of $t(q)$ and $\mathfrak{S}$, respectively, we find that if $\nu_{i}\left|\mathcal{B}_{0}, \delta_{i}\right| \mathcal{P}_{0}$ and $d_{i} \mid \mathcal{P}_{i}, i=1,2,3$, then

$$
\begin{aligned}
\mathfrak{S}\left(n ; Q ; \nu_{1} \delta_{1} d_{1}, \nu_{2} \delta_{2} d_{2}, \nu_{3} \delta_{3} d_{3}\right) & =8 \prod_{3 \leq p<Q_{0}}\left(1+t\left(p ; n ; \nu_{1}, \nu_{2}, \nu_{3}\right)\right) \prod_{Q_{0} \leq p<Q}\left(1+t\left(p ; n ; \delta_{1}, \delta_{2}, \delta_{3}\right)\right) .
\end{aligned}
$$

So, after some calculations we find that

$$
\Gamma_{1}^{\prime}=2 \pi \sum_{n \in \mathcal{F}}^{\#} \sqrt{n}\left(\prod_{3 \leq p<Q_{0}} \mathcal{V}_{p}(n)\right) \mathcal{H}^{+}(n) \mathcal{G}_{1}^{-} \mathcal{G}_{2}^{+} \mathcal{G}_{3}^{+},
$$

where

$$
\begin{aligned}
\mathcal{V}_{p}(n) & =\sum_{\nu_{1}, \nu_{2}, \nu_{3} \mid p} \frac{\mu\left(\nu_{1}\right) \mu\left(\nu_{2}\right) \mu\left(\nu_{3}\right)}{\varphi\left(\nu_{1}\right) \varphi\left(\nu_{2}\right) \varphi\left(\nu_{3}\right)}\left(1+t\left(p ; n ; \nu_{1}, \nu_{2}, \nu_{3}\right)\right), \\
\mathcal{H}^{ \pm}(n) & =\sum_{\delta_{1}, \delta_{2}, \delta_{3} \mid \mathcal{P}_{0}} \frac{\lambda_{0}^{ \pm}\left(\delta_{1}\right) \lambda_{0}^{+}\left(\delta_{2}\right) \lambda_{0}^{+}\left(\delta_{3}\right)}{\varphi\left(\delta_{1}\right) \varphi\left(\delta_{2}\right) \varphi\left(\delta_{3}\right)} \prod_{Q_{0} \leq p<Q}\left(1+t\left(p ; n ; \delta_{1}, \delta_{2}, \delta_{3}\right)\right), \\
\mathcal{G}_{i}^{ \pm} & =\sum_{d \mid \mathcal{P}_{i}} \frac{\lambda_{i}^{ \pm}(d)}{\varphi(d)}, \quad i=1,2,3 .
\end{aligned}
$$

We treat the sums $\Gamma_{i}, 2 \leq i \leq 7$, in the same manner and we find formulas similar to (3.11)-(3.13). Then we apply (3.10) to get

$$
\begin{aligned}
\Gamma \geq 2 & \pi \sum_{n \in \mathcal{F}}^{\#} \sqrt{n}\left(\prod_{3 \leq p<Q_{0}} \mathcal{V}_{p}(n)\right) \\
& \times\left(\mathcal{H}^{+}(n)\left(\mathcal{G}_{1}^{-} \mathcal{G}_{2}^{+} \mathcal{G}_{3}^{+}+\mathcal{G}_{1}^{+} \mathcal{G}_{2}^{-} \mathcal{G}_{3}^{+}+\mathcal{G}_{1}^{+} \mathcal{G}_{2}^{+} \mathcal{G}_{3}^{-}-5 \mathcal{G}_{1}^{+} \mathcal{G}_{2}^{+} \mathcal{G}_{3}^{+}\right)\right. \\
& \left.+3 \mathcal{H}^{-}(n) \mathcal{G}_{1}^{+} \mathcal{G}_{2}^{+} \mathcal{G}_{3}^{+}\right)+\mathcal{O}\left(X^{3} \mathcal{L}^{-A}\right) .
\end{aligned}
$$

Using (2.7), (2.8) we establish that

$$
\mathcal{V}_{p}(n)=\frac{p}{(p-1)^{3}} \sum_{\substack{1 \leq m_{1}, m_{2}, m_{3} \leq p-1 \\ m_{1}, m_{2}, m_{3} \neq p-2 \\ m_{1}^{2}+m_{2}^{2}+m_{3}^{2} \equiv n(p)}} 1 .
$$

This formula gives $0.001 \leq \mathcal{V}_{p}(n) \leq 3$ for $p=3,5,7$ and 11 . By the definition of $\mathcal{V}_{p}(n)$ and (2.14) we find another expression:

$$
\mathcal{V}_{p}(n)=1+h_{0}(p)-3 \frac{1+h_{1}(p)}{p-1}+3 \frac{1+h_{2}(p)}{(p-1)^{2}}-\frac{1+h_{3}(p)}{(p-1)^{3}}
$$


Using this formula and (2.10)-(2.13) we find that $1-9(p-1)^{-1} \leq \mathcal{V}_{p}(n) \leq$ $1+9(p-1)^{-1}$ for $p>11$. From the observations above and the definition (3.1) of $Q_{0}$ we obtain

$$
(\log \mathcal{L})^{-9} \ll \prod_{3 \leq p<Q_{0}} \mathcal{V}_{p}(n) \ll(\log \mathcal{L})^{9} .
$$

We leave the computations to the reader.

Consider the other quantities included in formula (3.14). Obviously

$$
\mathcal{G}_{i}^{ \pm} \ll \mathcal{L}, \quad i=1,2,3 .
$$

We have $\log D_{0} / \log Q \rightarrow \infty$ as $X \rightarrow \infty$. Hence we may expect that the sums $\mathcal{H}^{ \pm}(n)$ can be approximated by

$$
\mathcal{H}_{0}(n)=\sum_{\delta_{1}, \delta_{2}, \delta_{3} \mid \mathcal{P}_{0}} \frac{\mu\left(\delta_{1}\right) \mu\left(\delta_{2}\right) \mu\left(\delta_{3}\right)}{\varphi\left(\delta_{1}\right) \varphi\left(\delta_{2}\right) \varphi\left(\delta_{3}\right)} \prod_{Q_{0} \leq p<Q}\left(1+t\left(p ; n ; \delta_{1}, \delta_{2}, \delta_{3}\right)\right) .
$$

More precisely, we will prove that uniformly for $n \in \mathcal{F}$ satisfying $\nu(n-4) \leq$ $A \log \mathcal{L}$ the following formula holds:

$$
\mathcal{H}^{ \pm}(n)=\mathcal{H}_{0}(n)+\mathcal{O}\left(\mathcal{L}^{-2 A}\right) .
$$

We present the proof of (3.17) at the end of this section.

The sum $\mathcal{H}_{0}(n)$ is much more easy to deal with. We use (2.8)-(2.14) and after some elementary considerations we represent it as a product:

$$
\mathcal{H}_{0}(n)=\prod_{\substack{Q_{0} \leq p<Q \\ p \nmid \mathcal{P}_{0}}}\left(1+h_{0}(p)\right) \prod_{p \mid \mathcal{P}_{0}} \mathcal{V}_{p}(n)
$$

Now we are able to verify that

$$
(\log \mathcal{L})^{-14} \ll \mathcal{H}_{0}(n) \ll(\log \mathcal{L})^{14}
$$

Using (3.14)-(3.17) we get

$$
\Gamma \geq 2 \pi \sum_{n \in \mathcal{F}}^{\#} \sqrt{n}\left(\prod_{3 \leq p<Q_{0}} \mathcal{V}_{p}(n)\right) \mathcal{H}_{0}(n) \mathfrak{N}+\mathcal{O}\left(X^{3} \mathcal{L}^{-A}\right),
$$

where

$$
\mathfrak{N}=\mathcal{G}_{1}^{-} \mathcal{G}_{2}^{+} \mathcal{G}_{3}^{+}+\mathcal{G}_{1}^{+} \mathcal{G}_{2}^{-} \mathcal{G}_{3}^{+}+\mathcal{G}_{1}^{+} \mathcal{G}_{2}^{+} \mathcal{G}_{3}^{-}-2 \mathcal{G}_{1}^{+} \mathcal{G}_{2}^{+} \mathcal{G}_{3}^{+} .
$$

Arguing as in Section 8 of [23] we get

$$
\mathfrak{N} \gg(\log \mathcal{L})^{3} \mathcal{L}^{-3} .
$$

Therefore using (3.15), (3.18)-(3.20) we find that

$$
\Gamma \geq \mathcal{L}^{-5} \sum_{n \in \mathcal{F}}^{\#} \sqrt{n}+\mathcal{O}\left(X^{3} \mathcal{L}^{-A}\right)
$$


We combine the last estimate with (3.4) to obtain

$$
\sum_{n \in \mathcal{F}}^{\#} \sqrt{n} \ll X^{3} \mathcal{L}^{5-A} .
$$

Denote by $\mathcal{Y}^{\#}(N)$ the cardinality of the set $\{n \in \mathcal{F}: \nu(n-4) \leq A \log \mathcal{L}\}$. From the last formula we get

$$
\mathcal{Y}^{\#}(N) \ll X^{2} \mathcal{L}^{5-A / 2} .
$$

It remains to notice that $\mathcal{Y}(N)-\mathcal{Y}^{\#}(N) \ll X^{2} \mathcal{L}^{-A \log A+A-1}$ (see Hall and Tenenbaum [5], Chapter 0, for example). Therefore

$$
\mathcal{Y}(N) \ll X^{2} \mathcal{L}^{5-A / 2} .
$$

This proves Theorem 1.

It remains to establish the asymptotic formula (3.17). Consider, for example, the sum $\mathcal{H}^{+}(n)$. We have

$$
\mathcal{H}^{+}(n)=\mathcal{H}^{\prime}+\mathcal{H}^{\prime \prime}
$$

where in $\mathcal{H}^{\prime}$ we sum over $\delta_{1}, \delta_{2}, \delta_{3}$ such that $\left(\delta_{1}, \delta_{2}\right),\left(\delta_{1}, \delta_{3}\right),\left(\delta_{2}, \delta_{3}\right) \leq \mathcal{L}^{6 A}$. The sum $\mathcal{H}^{\prime \prime}$ is the contribution from the other summands. Using (2.10)(2.14) we may easily estimate the product from the formula for $\mathcal{H}^{+}(n)$ to get

$$
\mathcal{H}^{\prime \prime} \ll \mathcal{L} \sum_{\substack{\delta_{1}, \delta_{2}, \delta_{3} \mid \mathcal{P}_{0} \\\left(\delta_{1}, \delta_{2}\right)>\mathcal{L}^{6 A}}} \frac{\mu^{2}\left(\delta_{1}\right) \mu^{2}\left(\delta_{2}\right) \mu^{2}\left(\delta_{3}\right)}{\varphi\left(\delta_{1}\right) \varphi\left(\delta_{2}\right) \varphi\left(\delta_{3}\right)} \tau^{4}\left(\delta_{1}\right) \tau^{4}\left(\delta_{2}\right) \tau^{4}\left(\delta_{3}\right)\left(\delta_{1}, \delta_{2}, \delta_{3}\right) .
$$

After some standard calculations, which we leave to the reader, we find that

$$
\mathcal{H}^{\prime \prime} \ll \mathcal{L}^{-2 A} \text {. }
$$

Consider now $\mathcal{H}^{\prime}$. We have $\prod_{Q_{0} \leq p<Q}(1+t(p))=\Pi_{0} \Pi_{1} \Pi_{2} \Pi_{3}$, where $\Pi_{\nu}$ denotes the product of the primes dividing exactly $\nu$ of the integers $\delta_{1}, \delta_{2}, \delta_{3}$. It is clear that $\Pi_{2}$ and $\Pi_{3}$ are actually functions of $\left(\delta_{2}, \delta_{3}\right),\left(\delta_{1}, \delta_{3}\right),\left(\delta_{1}, \delta_{2}\right)$. Consider $\Pi_{0}$ and $\Pi_{1}$. For $h_{0}(p)$ defined by (2.10), we have $1+h_{0}(p)>0$ for any prime $p \geq Q_{0}$. The product $\mathcal{P}_{0}$ defined by (3.2) does not contain prime factors $p>2$ such that $p \mid n-4$ and $p \equiv 3(\bmod 4)$. Hence for any $p \mid \mathcal{P}_{0}$ we also have $1+h_{1}(p)>0$. We use the inclusion-exclusion principle and find that

$$
\Pi_{0} \Pi_{1}=\xi(n) \prod_{p \mid \delta_{1}} \frac{1+h_{1}(p)}{1+h_{0}(p)} \prod_{p \mid \delta_{2}} \frac{1+h_{1}(p)}{1+h_{0}(p)} \prod_{p \mid \delta_{3}} \frac{1+h_{1}(p)}{1+h_{0}(p)} \Pi^{\prime}
$$

where

$$
\xi(n)=\prod_{Q_{0} \leq p<Q}\left(1+h_{0}(p)\right)
$$


and where $\Pi^{\prime}$ is actually a function of $\left(\delta_{2}, \delta_{3}\right),\left(\delta_{1}, \delta_{3}\right),\left(\delta_{1}, \delta_{2}\right)$. So we may write

$$
\mathcal{H}^{\prime}=\xi(n) \sum_{\substack{\delta_{1}, \delta_{2}, \delta_{3} \mid \mathcal{P}_{0} \\\left(\delta_{i}, \delta_{j} \leq \mathcal{L}^{6 A} \\ 1 \leq i<j \leq 3\right.}} \kappa\left(\left(\delta_{2}, \delta_{3}\right),\left(\delta_{1}, \delta_{3}\right),\left(\delta_{1}, \delta_{2}\right)\right) \prod_{\nu=1}^{3}\left(\lambda_{0}^{+}\left(\delta_{\nu}\right) \frac{\omega\left(\delta_{\nu}\right)}{\delta_{\nu}}\right)
$$

where $\kappa$ arises from $\Pi^{\prime}, \Pi_{2}, \Pi_{3}$ and where

$$
\omega(k)= \begin{cases}\frac{k}{\varphi(k)} \prod_{p \mid k} \frac{1+h_{1}(p)}{1+h_{0}(p)} & \text { if }\left(k, 2 \mathcal{B}_{0}\right)=1, \\ 0 & \text { otherwise. }\end{cases}
$$

We may easily find an explicit formula for $\kappa\left(l_{1}, l_{2}, l_{3}\right)$. Then we use $(2.10)-$ (2.13) to find that

$$
\kappa\left(l_{1}, l_{2}, l_{3}\right) \ll\left(l_{1} l_{2} l_{3}\right)^{10} .
$$

In fact, a much sharper estimate is available. We leave the calculations to the reader.

We use (3.24) to represent $\mathcal{H}^{\prime}$ as follows:

$$
\begin{aligned}
& \mathcal{H}^{\prime}=\xi(n) \sum_{\substack{l_{1}, l_{2}, l_{3} \mid \mathcal{P}_{0} \\
l_{1}, l_{2}, l_{3} \leq \mathcal{L}^{6 A}}} \kappa\left(l_{1}, l_{2}, l_{3}\right) \\
& \times \sum_{\substack{\delta_{1}, \delta_{2}, \delta_{3} \mid \mathcal{P}_{0} \\
\left(\delta_{2}, \delta_{3}\right)=l_{1},\left(\delta_{1}, \delta_{3}\right)=l_{2} \\
\left(\delta_{1}, \delta_{2}\right)=l_{3}}} \prod_{\nu=1}^{3}\left(\lambda_{0}^{+}\left(\delta_{\nu}\right) \frac{\omega\left(\delta_{\nu}\right)}{\delta_{\nu}}\right) \\
& =\xi(n) \sum_{\substack{l_{1}, l_{2}, l_{3} \mid \mathcal{P}_{0} \\
l_{1}, l_{2}, l_{3} \leq \mathcal{L}^{6 A}}} \kappa\left(l_{1}, l_{2}, l_{3}\right) \\
& \times \quad \sum_{\delta_{1}, \delta_{2}, \delta_{3} \mid \mathcal{P}_{0}} \prod_{\nu=1}^{3}\left(\lambda_{0}^{+}\left(\delta_{\nu}\right) \frac{\omega\left(\delta_{\nu}\right)}{\delta_{\nu}}\right) \\
& \delta_{1} \equiv 0\left(\left[l_{2}, l_{3}\right]\right), \delta_{2} \equiv 0\left(\left[l_{1}, l_{3}\right]\right) \\
& \delta_{3} \equiv 0\left(\left[l_{1}, l_{2}\right]\right) \\
& \times \sum_{h_{1} \mid\left(\delta_{2} / l_{1}, \delta_{3} / l_{1}\right)} \mu\left(h_{1}\right) \sum_{h_{2} \mid\left(\delta_{1} / l_{2}, \delta_{3} / l_{2}\right)} \mu\left(h_{2}\right) \sum_{h_{3} \mid\left(\delta_{1} / l_{3}, \delta_{2} / l_{3}\right)} \mu\left(h_{3}\right) \\
& =\xi(n) \sum_{\substack{l_{1}, l_{2}, l_{3} \mid \mathcal{P}_{0} \\
l_{1}, l_{2}, l_{3} \leq \mathcal{L}^{6 A}}} \kappa\left(l_{1}, l_{2}, l_{3}\right) \sum_{\substack{h_{i} \mid \mathcal{P}_{0} / l_{i} \\
i=1,2,3}} \mu\left(h_{1}\right) \mu\left(h_{2}\right) \mu\left(h_{3}\right) \mathcal{D}_{1} \mathcal{D}_{2} \mathcal{D}_{3},
\end{aligned}
$$


where

$$
\mathcal{D}_{i}=\sum_{\substack{\delta \mid \mathcal{P}_{0} \\ \delta \equiv 0\left(\varrho_{i}\right)}} \lambda_{0}^{+}(\delta) \frac{\omega(\delta)}{\delta}, \quad i=1,2,3
$$

and

$$
\varrho_{1}=\left[l_{2} h_{2}, l_{3} h_{3}\right], \quad \varrho_{2}=\left[l_{1} h_{1}, l_{3} h_{3}\right], \quad \varrho_{3}=\left[l_{1} h_{1}, l_{2} h_{2}\right] .
$$

It is not difficult to see that the function $\omega(k)$ defined by $(3.25)$ satisfies

$$
\prod_{w_{1} \leq p<w_{2}}\left(1-\frac{\omega(p)}{p}\right)^{-1} \leq \frac{\log w_{2}}{\log w_{1}}\left(1+\frac{c}{\log w_{1}}\right),
$$

for some constant $c>0$ and for arbitrary $2 \leq w_{1}<w_{2}$. Only at this point do we use the fact that the integers $n$ satisfy $\nu(n-4) \leq A \log \mathcal{L}$. We note that $\log D_{0} / \log Q \geq \sqrt{\mathcal{L}}$. Therefore we may use Lemma 11 of [1] to get

$$
\mathcal{D}_{i}=\mathcal{E}_{i}+\mathcal{O}\left(\tau\left(\varrho_{i}\right) \exp (-\sqrt{\mathcal{L}})\right), \quad i=1,2,3,
$$

where

$$
\mathcal{E}_{i}=\sum_{\substack{\delta \mid \mathcal{P}_{0} \\ \delta \equiv 0\left(\varrho_{i}\right)}} \mu(\delta) \frac{\omega(\delta)}{\delta}, \quad i=1,2,3 .
$$

It is also easy to see that the sums $\mathcal{D}_{i}$ and $\mathcal{E}_{i}$ defined above satisfy

$$
\left|\mathcal{D}_{i}\right|,\left|\mathcal{E}_{i}\right| \ll \mu^{2}\left(\varrho_{i}\right) \tau^{3}\left(\varrho_{i}\right) \varrho_{i}^{-1} \mathcal{L} .
$$

We replace the product $\mathcal{D}_{1} \mathcal{D}_{2} \mathcal{D}_{3}$ from $(3.27)$ by $\mathcal{E}_{1} \mathcal{E}_{2} \mathcal{E}_{3}$ and denote the new sum by $\mathcal{H}^{*}$. Proceeding as in Section 7 of [23] and using (3.23), (3.26)-(3.30) we get

$$
\mathcal{H}^{\prime}=\mathcal{H}^{*}+\mathcal{O}\left(\mathcal{L}^{-2 A}\right)
$$

To study $\mathcal{H}^{*}$ we apply the procedures above in reverse order and we obtain

$$
\mathcal{H}^{*}=\mathcal{H}_{0}(n)+\mathcal{O}\left(\mathcal{L}^{-2 A}\right) .
$$

Formula (3.17) for $\mathcal{H}^{+}(n)$ is a consequence of (3.21), (3.22), (3.31) and $(3.32)$.

4. Proof of the Proposition-minor arcs. The object of this section is to prove the inequality $(2.23)$ for $\mathcal{U}_{2}$. We substitute the expression for $I_{2}$, given by (2.6), in formula (2.21) and change the order of summation and integration to obtain

$$
\mathcal{U}_{2}=\sum_{n \leq N}\left|\int_{E_{2}} \mathcal{K}_{1}(\alpha) \mathcal{K}_{2}(\alpha) \mathcal{K}_{3}(\alpha) e(-n \alpha) d \alpha\right|,
$$

where 


$$
\mathcal{K}_{i}(\alpha)=\sum_{k \leq K_{i}} \beta_{i}(k) S_{k}(\alpha), \quad i=1,2,3
$$

We apply the Cauchy and Bessel inequalities to get

$$
\begin{aligned}
\mathcal{U}_{2}^{2} & \ll N \sum_{n \leq N}\left|\int_{E_{2}} \mathcal{K}_{1}(\alpha) \mathcal{K}_{2}(\alpha) \mathcal{K}_{3}(\alpha) e(-n \alpha) d \alpha\right|^{2} \\
& \ll N \int_{E_{2}}\left|\mathcal{K}_{1}(\alpha) \mathcal{K}_{2}(\alpha) \mathcal{K}_{3}(\alpha)\right|^{2} d \alpha \\
& \ll N\left(\max _{\alpha \in E_{2}}\left|\mathcal{K}_{3}(\alpha)\right|\right)^{2} \int_{0}^{1}\left|\mathcal{K}_{1}(\alpha) \mathcal{K}_{2}(\alpha)\right|^{2} d \alpha \\
& \ll N\left(\max _{\alpha \in E_{2}}\left|\mathcal{K}_{3}(\alpha)\right|\right)^{2}\left(\int_{0}^{1}\left|\mathcal{K}_{1}(\alpha)\right|^{4} d \alpha+\int_{0}^{1}\left|\mathcal{K}_{2}(\alpha)\right|^{4} d \alpha\right) .
\end{aligned}
$$

To estimate the last expression we prove the inequalities

$$
\int_{0}^{1}\left|\mathcal{K}_{i}(\alpha)\right|^{4} d \alpha \ll X^{2} \mathcal{L}^{1027}, \quad i=1,2,
$$

and

$$
\max _{\alpha \in E_{2}}\left|\mathcal{K}_{3}(\alpha)\right| \ll X \mathcal{L}^{-2 A} .
$$

Formula (2.23) for $\mathcal{U}_{2}$ is a consequence of (4.2)-(4.4).

First we prove (4.3). Denote the integral on the left-hand side of (4.3) by $\mathfrak{I}$. We use $(2.2),(2.18)$ and (4.1) to get

$$
\begin{aligned}
& \mathfrak{I}=\int_{0}^{1} \sum_{k_{1}, \ldots, k_{4} \leq K_{i}} \beta_{i}\left(k_{1}\right) \beta_{i}\left(k_{2}\right) \overline{\beta_{i}\left(k_{3}\right) \beta_{i}\left(k_{4}\right)} S_{k_{1}}(\alpha) S_{k_{2}}(\alpha) S_{k_{3}}(-\alpha) S_{k_{4}}(-\alpha) d \alpha \\
& =\sum_{k_{1}, \ldots, k_{4} \leq K_{i}} \beta_{i}\left(k_{1}\right) \beta_{i}\left(k_{2}\right) \overline{\beta_{i}\left(k_{3}\right) \beta_{i}\left(k_{4}\right)} \\
& \times \int_{0}^{1} \sum_{\substack{p_{1}, \ldots, p_{4} \leq X \\
p_{j}+2 \equiv 0}}\left(\log p_{1}\right) \ldots\left(\log p_{4}\right) e\left(\alpha\left(p_{1}^{2}+p_{2}^{2}-p_{3}^{2}-p_{4}^{2}\right)\right) d \alpha \\
& =\sum_{k_{1}, \ldots, k_{4} \leq K_{i}} \beta_{i}\left(k_{1}\right) \beta_{i}\left(k_{2}\right) \overline{\beta_{i}\left(k_{3}\right) \beta_{i}\left(k_{4}\right)} \\
& \times \sum_{\substack{p_{1}, \ldots, p_{4} \leq X \\
p_{j}+2 \equiv=0\left(k_{j}\right), 1 \leq j \leq 4 \\
p_{1}^{2}+p_{2}^{2}=p_{3}^{2}+p_{4}^{2}}}\left(\log p_{1}\right) \ldots\left(\log p_{4}\right)
\end{aligned}
$$




$$
\begin{aligned}
& \ll \mathcal{L}^{4} \sum_{k_{1}, \ldots, k_{4} \leq K_{i}} \tau\left(k_{1}\right) \ldots \tau\left(k_{4}\right) \sum_{\begin{array}{c}
n_{1}, \ldots, n_{4} \leq X \\
n_{j}+2 \equiv 0\left(k_{j}\right), 1 \leq j \leq 4 \\
n_{1}^{2}+n_{2}^{2}=n_{3}^{2}+n_{4}^{2}
\end{array}} 1 \\
& =\mathcal{L}^{4} \sum_{\substack{n_{1}, \ldots, n_{4} \leq X \\
n_{1}^{2}+n_{2}^{2}=n_{3}^{2}+n_{4}^{2}}}\left(\sum_{\substack{k_{1} \leq K_{i} \\
k_{1} \mid n_{1}+2}} \tau\left(k_{1}\right)\right) \ldots\left(\sum_{\substack{k_{4} \leq K_{i} \\
k_{4} \mid n_{4}+2}} \tau\left(k_{4}\right)\right) \\
& \ll \mathcal{L}^{4} \sum_{\substack{n_{1}, \ldots, n_{4} \leq X \\
n_{1}^{2}+n_{2}^{2}=n_{3}^{2}+n_{4}^{2}}} \tau^{2}\left(n_{1}+2\right) \tau^{2}\left(n_{2}+2\right) \tau^{2}\left(n_{3}+2\right) \tau^{2}\left(n_{4}+2\right) .
\end{aligned}
$$

To estimate the last sum we apply the inequality $x y z t \leq x^{4}+y^{4}+z^{4}+t^{4}$. Then we split the new sum into two parts to obtain

$$
\mathfrak{I} \ll \mathcal{L}^{4} \sum_{\substack{n_{1}, \ldots, n_{4} \leq X \\ n_{1}^{2}+n_{2}^{2}=n_{3}^{2}+n_{4}^{2}}} \tau^{8}\left(n_{1}+2\right) \ll X^{2} \mathcal{L}^{259}+\mathcal{L}^{4} U_{0},
$$

where

$$
U_{0}=\sum_{\substack{n_{1}, \ldots, n_{4} \leq X \\\left(n_{1}-n_{3}\right)\left(n_{1}+n_{3}\right)=\left(n_{4}-n_{2}\right)\left(n_{4}+n_{2}\right) \\ n_{1} \neq n_{3}, n_{1} \neq n_{4}, n_{2} \neq n_{3}, n_{2} \neq n_{4}}} \tau^{8}\left(n_{1}+2\right) .
$$

We divide $U_{0}$ into two subsums:

$$
U_{0}=U_{1}+U_{2}
$$

In the domain of summation of $U_{1}$ the condition $n_{1} \neq n_{3}$ is replaced by $n_{1}>n_{3}$, in $U_{2}$ it is replaced by $n_{1}<n_{3}$.

Consider $U_{1}$. We have

$$
\begin{aligned}
& U_{1}=\sum_{\substack{h_{1}, \ldots, h_{4} \leq 2 X \\
h_{1} h_{3}=h_{2} h_{4} \\
h_{1} \equiv h_{3}(2), h_{2} \equiv h_{4}(2)}} \sum_{\begin{array}{c}
n_{1}, \ldots, n_{4} \leq X \\
n_{1}-n_{3}=h_{1}, n_{1}+n_{3}=h_{3} \\
n_{4}-n_{2}=h_{2}, n_{4}+n_{2}=h_{4}
\end{array}} \tau^{8}\left(n_{1}+2\right) \\
& \ll \sum_{\substack{h_{1}, \ldots, h_{4} \leq 2 X \\
h_{1} h_{3}=h_{2} h_{4}}} \tau^{8}\left(h_{1}+h_{3}+4\right) \\
& =\sum_{k, l \leq 2 X} \sum_{\begin{array}{c}
h_{1}, \ldots, h_{4} \leq 2 X \\
h_{1}, h_{3}=h_{2} h_{4} \\
\left(h_{1}, h_{2}\right)=k,\left(h_{3}, h_{4}\right)=l
\end{array}} \tau^{8}\left(h_{1}+h_{3}+4\right) \\
& \ll \sum_{k, l \leq 2 X} \sum_{\substack{h_{1}, h_{2} \leq(2 X) / k ; h_{3}, h_{4} \leq(2 X) / l \\
h_{1} h_{3}=h_{2} h_{4} \\
\left(h_{1}, h_{2}\right)=\left(h_{3}, h_{4}\right)=1}} \tau^{8}\left(h_{1} k+h_{3} l+4\right) .
\end{aligned}
$$


The conditions $\left(h_{1}, h_{2}\right)=\left(h_{3}, h_{4}\right)=1, h_{1} h_{3}=h_{2} h_{4}$ imply $h_{1}=h_{4}, h_{2}=h_{3}$. Hence

$$
\begin{aligned}
U_{1} & \ll \sum_{k, l \leq 2 X} \sum_{\substack{h_{1}, h_{2} \leq \min ((2 X) / k,(2 X) / l) \\
m_{1}, m_{2} \leq 2 X}} \tau^{8}\left(h_{1} k+h_{2} l+4\right) \\
& \ll \sum_{\substack{k, l \leq 2 X \\
h_{1}, h_{2} \leq \min ((2 X) / k,(2 X) / l) \\
h_{1} k=m_{1}, h_{2} l=m_{2}}} 1 \\
& \ll \sum_{m_{1}, m_{2} \leq 2 X} \tau^{8}\left(m_{1}+m_{2}+4\right) \tau\left(m_{1}\right) \tau\left(m_{2}\right) .
\end{aligned}
$$

Now we apply the inequality $x^{8} y z \leq x^{10}+y^{10}+z^{10}$ to get

$$
\begin{aligned}
U_{1} & \ll \sum_{m_{1}, m_{2} \leq 2 X} \tau^{10}\left(m_{1}+m_{2}+4\right)+\sum_{m_{1}, m_{2} \leq 2 X} \tau^{10}\left(m_{1}\right) \\
& \ll \sum_{l \leq 4 X+4} \tau^{10}(l) \sum_{\substack{m_{1}, m_{2} \leq 2 X \\
m_{1}+m_{2}+4=l}} 1+X^{2} \mathcal{L}^{2^{10}-1} \ll X^{2} \mathcal{L}^{2^{10}-1} .
\end{aligned}
$$

We treat $U_{2}$ similarly to obtain

$$
U_{2} \ll X^{2} \mathcal{L}^{2^{10}-1} .
$$

The inequality (4.3) follows from (4.5), (4.7)-(4.9).

Let us now prove (4.4). For simplicity we write $K$ and $\beta(k)$ instead of $K_{3}$ and $\beta_{3}(k)$, respectively. We decompose $\mathcal{K}_{3}(\alpha)$ into $\mathcal{O}(\mathcal{L})$ sums of the form

$$
\mathcal{K}(\alpha, Y)=\sum_{k \leq K} \beta(k) \sum_{\substack{Y<p \leq 2 Y \\ p+2 \equiv 0(k)}} \log p e(\alpha p) .
$$

We may assume that $X \mathcal{L}^{-2 A-4}<Y \leq X / 2$, for otherwise we can use the trivial estimate for $\mathcal{K}(\alpha, Y)$. We have

$$
\mathcal{K}(\alpha, Y)=W(Y, K, \alpha)+\mathcal{O}\left(X^{2 / 3}\right),
$$

where

$$
W(Y, K, \alpha)=\sum_{Y<n \leq 2 Y} \Lambda(n) e\left(\alpha n^{2}\right) \sum_{\substack{k \leq K \\ k \mid n+2}} \beta(k) .
$$

We apply Heath-Brown's identity [7] to decompose $W(Y, K, \alpha)$ into $\mathcal{O}\left(\mathcal{L}^{6}\right)$ sums of two types.

Type I sums are

$$
W_{1}=\sum_{\substack{M<m \leq M_{1} \\ Y<m l \leq 2 Y}} \sum_{\substack{L<l \leq L_{1} \\ a_{m}}} a_{\left(\alpha m^{2} l^{2}\right)} \sum_{\substack{k \leq K \\ k \mid m l+2}} \beta(k)
$$

and 


$$
W_{1}^{\prime}=\sum_{\substack{M<m \leq M_{1} \\ Y<m l \leq 2 Y}} \sum_{\substack{L<l \leq L_{1} \\ Y<m}} a_{m}(\log l) e\left(\alpha m^{2} l^{2}\right) \sum_{\substack{k \leq K \\ k \mid m l+2}} \beta(k),
$$

where

(4.11) $M_{1} \leq 2 M, \quad L_{1} \leq 2 L, \quad M L \asymp Y, \quad L \geq Y^{0.498}, \quad\left|a_{m}\right| \ll \tau_{5}(m) \mathcal{L}$.

Type II sums are

$$
W_{2}=\sum_{\substack{M<m \leq M_{1} \\ Y<m l \leq 2 Y}} \sum_{\substack{L<l \leq L_{1} \\ Y<m}} a_{m} b_{l} e\left(\alpha m^{2} l^{2}\right) \sum_{\substack{k \leq K \\ k \mid m l+2}} \beta(k),
$$

where

$$
\begin{gathered}
M_{1} \leq 2 M, \quad L_{1} \leq 2 L, \quad M L \asymp Y, \quad Y^{0.001} \leq L \leq 2^{30} Y^{1 / 3}, \\
\left|a_{m}\right| \ll \tau_{5}(m) \mathcal{L}, \quad\left|b_{l}\right| \ll \tau_{5}(l) \mathcal{L} .
\end{gathered}
$$

Consider type II sums. We have

$$
\left|W_{2}\right| \ll \mathcal{L} \sum_{M<m \leq M_{1}} \tau_{5}(m)\left|\sum_{\substack{L<l \leq L_{1} \\ Y<m l \leq 2 Y \\ m l+2 \equiv 0(k)}} \sum_{\substack{k \leq K \\ m l}} b_{l} \beta(k) e\left(\alpha m^{2} l^{2}\right)\right| .
$$

An application of Cauchy's inequality gives

$$
\begin{gathered}
\left|W_{2}\right|^{2} \ll M \mathcal{L}^{26} \sum_{M<m \leq M_{1}}\left|\sum_{\substack{L<l \leq L_{1} \\
Y<m l \leq 2 Y \\
m l+2 \equiv 0(k)}} \sum_{\substack{k \leq K \\
l+2}} b_{l} \beta(k) e\left(\alpha m^{2} l^{2}\right)\right|^{2} \\
=M \mathcal{L}^{26} \sum_{M<m \leq M_{1}} \sum_{\substack{L<l_{1}, l_{2} \leq L_{1} \\
Y<l_{1} m, l_{2} m \leq 2 Y \\
l_{i} m+2 \equiv 0\left(k_{i}\right), i=1,2}} \sum_{k_{1}, k_{2} \leq K} b_{l_{1}} \bar{b}_{l_{2}} \\
\times \beta\left(k_{1}\right) \overline{\beta\left(k_{2}\right)} e\left(\alpha m^{2}\left(l_{1}^{2}-l_{2}^{2}\right)\right) .
\end{gathered}
$$

Therefore, by (2.18) and (4.12),

$$
\left|W_{2}\right|^{2} \ll M \mathcal{L}^{28} \sum_{\begin{array}{c}
k_{1}, k_{2} \leq K \\
\left(k_{1} k_{2}, 2\right)=\left(l_{1}, k_{1}\right)=\left(l_{2}, k_{2}\right)=1
\end{array}} \sum_{\substack{L<l_{1}, l_{2} \leq L_{1} \\
l_{1}=l_{2}\left(\left(k_{1}, k_{2}\right)\right)}} \tau\left(k_{1}\right) \tau\left(k_{2}\right) \tau_{5}\left(l_{1}\right) \tau_{5}\left(l_{2}\right)|V|,
$$

where

$$
V=\sum_{\substack{M^{\prime}<m \leq M_{1}^{\prime} \\ l_{i} m+2 \equiv 0\left(k_{i}\right), i=1,2}} e\left(\alpha m^{2}\left(l_{1}^{2}-l_{2}^{2}\right)\right),
$$

$$
M^{\prime}=\max \left(Y / l_{1}, Y / l_{2}, M\right), \quad M_{1}^{\prime}=\min \left(2 Y / l_{1}, 2 Y / l_{2}, M_{1}\right) .
$$

Note that if $l_{1} \not \equiv l_{2}\left(\left(k_{1}, k_{2}\right)\right)$ then the system of congruences $l_{i} m+2 \equiv 0\left(k_{i}\right)$, $i=1,2$, is not solvable and, therefore, $V=0$. Using only the basic properties 
of the congruences we easily find that if the conditions imposed on $l_{i}, k_{i}$ in (4.13) hold, then there exists some integer $h_{0}=h_{0}\left(l_{1}, l_{2}, k_{1}, k_{2}\right)$ satisfying $1 \leq h_{0} \leq\left[k_{1}, k_{2}\right]$ and such that the system $l_{i} m+2 \equiv 0\left(k_{i}\right), i=1,2$, is equivalent to the congruence $m \equiv h_{0}\left(\left[k_{1}, k_{2}\right]\right)$. In this case we have

$$
\begin{aligned}
|V| & =\left|\sum_{\substack{M^{\prime}<m \leq M_{1}^{\prime} \\
m \equiv h_{0}\left(\left[k_{1}, k_{2}\right]\right)}} e\left(\alpha m^{2}\left(l_{1}^{2}-l_{2}^{2}\right)\right)\right| \\
& =\left|\sum_{\substack{H<r \leq H_{1}\\
}} e\left(\alpha\left(h_{0}+r\left[k_{1}, k_{2}\right]\right)^{2}\left(l_{1}^{2}-l_{2}^{2}\right)\right)\right| \\
& =\left|\sum_{H<r \leq H_{1}} e\left(\alpha\left(r^{2}\left[k_{1}, k_{2}\right]^{2}+2 h_{0} r\left[k_{1}, k_{2}\right]\right)\left(l_{1}^{2}-l_{2}^{2}\right)\right)\right|,
\end{aligned}
$$

where

$$
H=\frac{M^{\prime}-h_{0}}{\left[k_{1}, k_{2}\right]}, \quad H_{1}=\frac{M_{1}^{\prime}-h_{0}}{\left[k_{1}, k_{2}\right]} .
$$

The trivial estimate for the sum $V$ is

$$
|V| \ll \frac{M}{\left[k_{1}, k_{2}\right]} .
$$

Note that, according to (2.17), (4.12) and our assumption $Y>X \mathcal{L}^{-2 A-4}$, we have $\left[k_{1}, k_{2}\right] \ll M \mathcal{L}^{-200 A}$. If the upper bound for $K$ given by (4.3) were greater, for example $X^{1 / 3+\varepsilon}$ for some $\varepsilon>0$, then our method would not work. Indeed, in this case the trivial estimate for $V$ would be $|V| \ll 1$ for some $k_{1}, k_{2}$ and it would be difficult to find a non-trivial estimate for the sum $W_{2}$.

We easily see that the contribution of the summands with $l_{1}=l_{2}$ in the expression on the right-hand side of (4.13) is

$$
\ll M^{2} \mathcal{L}^{28} \sum_{k_{1}, k_{2} \leq K} \frac{\tau\left(k_{1}\right) \tau\left(k_{2}\right)}{\left[k_{1}, k_{2}\right]} \sum_{L<l \leq L_{1}} \tau_{5}^{2}(l) \ll M^{2} L \mathcal{L}^{100} .
$$

By the last observation, Cauchy's inequality and the estimate (4.13) we get

$$
\begin{aligned}
& \left|W_{2}\right|^{4} \ll M^{4} L^{2} \mathcal{L}^{200} \\
& +M^{2} \mathcal{L}^{60}\left(\sum_{k_{1}, k_{2} \leq K} \frac{\tau^{2}\left(k_{1}\right) \tau^{2}\left(k_{2}\right)}{\left[k_{1}, k_{2}\right]} \sum_{L<l_{1}, l_{2} \leq L_{1}} \tau_{5}^{2}\left(l_{1}\right) \tau_{5}^{2}\left(l_{2}\right)\right) \\
& \times\left(\sum_{\substack{k_{1}, k_{2} \leq K \\
\left(k_{1} k_{2}, 2\right)=1}}\left[k_{1}, k_{2}\right] \sum_{\begin{array}{c}
L<l_{1}, l_{2} \leq L_{1}, l_{1} \neq l_{2} \\
\left(k_{1}, l_{1}\right)=\left(k_{2}, l_{2}\right)=1 \\
l_{1} \equiv l_{2}\left(\left(k_{1}, k_{2}\right)\right)
\end{array}}|V|^{2}\right) \\
& \ll M^{4} L^{2} \mathcal{L}^{200}+M^{2} L^{2} \mathcal{L}^{200} \Sigma_{0},
\end{aligned}
$$


where

$$
\begin{aligned}
\Sigma_{0}= & \sum_{\begin{array}{c}
k_{1}, k_{2} \leq K \\
\left(k_{1} k_{2}, 2\right)=1
\end{array}}\left[k_{1}, k_{2}\right] \sum_{\begin{array}{c}
L<l_{1}, l_{2} \leq L_{1}, l_{1} \neq l_{2} \\
\left(k_{1}, l_{1}\right)=\left(k_{2}, l_{2}\right)=1 \\
l_{1} \equiv l_{2}\left(\left(k_{1}, k_{2}\right)\right)
\end{array}} e\left(\alpha\left(\left(r_{1}^{2}-r_{2}^{2}\right)\left[k_{1}, k_{2}\right]^{2}+2 h_{0}\left[k_{1}, k_{2}\right]\left(r_{1}-r_{2}\right)\right)\left(l_{1}^{2}-l_{2}^{2}\right)\right) . \\
& \times \sum_{H<r_{1}, r_{2} \leq H_{1}} e(.)
\end{aligned}
$$

We have

$$
\begin{aligned}
& \Sigma_{0}=\sum_{\begin{array}{c}
k_{1}, k_{2} \leq K \\
\left(k_{1} k_{2}, 2\right)=1
\end{array}}\left[k_{1}, k_{2}\right] \sum_{\begin{array}{c}
L<l_{1}, l_{2} \leq L_{1}, l_{1} \neq l_{2} \\
\left(k_{1}, l_{1}\right)=\left(k_{2}, l_{2}\right)=1 \\
l_{1} \equiv l_{2}\left(\left(k_{1}, k_{2}\right)\right)
\end{array}} \\
& \times \sum_{s_{1}, s_{2}} e\left(\alpha\left(s_{1} s_{2}\left[k_{1}, k_{2}\right]^{2}+2 h_{0} s_{1}\left[k_{1}, k_{2}\right]\right)\left(l_{1}^{2}-l_{2}^{2}\right)\right) \sum_{\substack{t<r_{1}, r_{2} \leq H_{1} \\
r_{1}-r_{2}=s_{1} \\
r_{1}+r_{2}=s_{2}}} 1 \\
& =\sum_{\substack{k_{1}, k_{2} \leq K \\
\left(k_{1} k_{2}, 2\right)=1}}\left[k_{1}, k_{2}\right] \sum_{\substack{L<l_{1}, l_{2} \leq L_{1}, l_{1} \neq l_{2} \\
\left(k_{1}, l_{1}\right)=\left(k_{2}, l_{2}\right)=1 \\
l_{1} \equiv l_{2}\left(\left(k_{1}, k_{2}\right)\right)}} \\
& \times \sum_{\substack{s_{1}, s_{2}: s_{1} \equiv s_{2}(2) \\
2 H<s_{2}+s_{1} \leq 2 H_{1} \\
2 H<s_{2}-s_{1} \leq 2 H_{1}}} e\left(\alpha\left(s_{1} s_{2}\left[k_{1}, k_{2}\right]^{2}+2 h_{0} s_{1}\left[k_{1}, k_{2}\right]\right)\left(l_{1}^{2}-l_{2}^{2}\right)\right) \\
& =\sum_{\begin{array}{c}
k_{1}, k_{2} \leq K \\
\left(k_{1} k_{2}, 2\right)=1
\end{array}}\left[k_{1}, k_{2}\right] \sum_{\begin{array}{c}
L<l_{1}, l_{2} \leq L_{1}, l_{1} \neq l_{2} \\
\left(k_{1}, l_{1}\right)=\left(k_{2}, l_{2}\right)=1 \\
l_{1} \equiv l_{2}\left(\left(k_{1}, k_{2}\right)\right)
\end{array}} \\
& \times \sum_{\left|s_{1}\right| \leq 2 H_{1}-2 H} e\left(2 \alpha h_{0} s_{1}\left[k_{1}, k_{2}\right]\left(l_{1}^{2}-l_{2}^{2}\right)\right) \\
& \times \sum_{\substack{s_{2}: s_{2} \equiv s_{1}(2) \\
2 H-s_{1}<s_{2} \leq 2 H_{1}-s_{1} \\
2 H+s_{1}<s_{2} \leq 2 H_{1}+s_{1}}} e\left(\alpha s_{1} s_{2}\left[k_{1}, k_{2}\right]^{2}\left(l_{1}^{2}-l_{2}^{2}\right)\right) .
\end{aligned}
$$

Define

$$
K_{0}=\mathcal{L}^{50 A}
$$

We divide the sum $\Sigma_{0}$ into two parts:

$$
\Sigma_{0}=\Sigma_{1}+\Sigma_{2}
$$

In $\Sigma_{1}$ the restriction $\left[k_{1}, k_{2}\right] \leq K_{0}$ is imposed on the domain of summation over $k_{1}, k_{2}$, whilst in $\Sigma_{2}$ we sum over $k_{1}, k_{2}$ satisfying the condition $\left[k_{1}, k_{2}\right]>$ $K_{0}$. According to (4.17) and the definitions above, we put $s_{2}=s_{1}+2 t$ and 
obtain

$$
\begin{aligned}
& \Sigma_{1} \leq \sum_{\begin{array}{c}
k_{1}, k_{2} \leq K_{0} \\
\left(k_{1} k_{2}, 2\right)=1
\end{array}}\left[k_{1}, k_{2}\right] \sum_{\begin{array}{c}
L<l_{1}, l_{2} \leq L_{1}, l_{1} \neq l_{2} \\
\left(k_{1}, l_{1}\right)=\left(k_{2}, l_{2}\right)=1 \\
l_{1} \equiv l_{2}\left(\left(k_{1}, k_{2}\right)\right)
\end{array}} \\
& \times \sum_{\left|s_{1}\right| \leq 2 H_{1}-2 H}\left|\sum_{H^{\prime}<t \leq H_{1}^{\prime}} e\left(2 \alpha s_{1}\left[k_{1}, k_{2}\right]^{2}\left(l_{1}^{2}-l_{2}^{2}\right) t\right)\right|, \\
& \Sigma_{2} \leq \sum_{\begin{array}{c}
k_{1}, k_{2} \leq K \\
\left(k_{1} k_{2}, 2\right)=1 \\
{\left[k_{1}, k_{2}\right]>K_{0}}
\end{array}}\left[k_{1}, k_{2}\right] \sum_{\begin{array}{c}
L<l_{1}, l_{2} \leq L_{1}, l_{1} \neq l_{2} \\
\left(k_{1}, l_{1}\right)=\left(k_{2}, l_{2}\right)=1 \\
l_{1} \equiv l_{2}\left(\left(k_{1}, k_{2}\right)\right)
\end{array}} \\
& \times \sum_{\left|s_{1}\right| \leq 2 H_{1}-2 H}\left|\sum_{H^{\prime}<t \leq H_{1}^{\prime}} e\left(2 \alpha s_{1}\left[k_{1}, k_{2}\right]^{2}\left(l_{1}^{2}-l_{2}^{2}\right) t\right)\right| \text {, }
\end{aligned}
$$

where

$$
H^{\prime}=\max \left(H-s_{1}, H\right), \quad H_{1}^{\prime}=\min \left(H_{1}-s_{1}, H_{1}\right) .
$$

Consider first $\Sigma_{1}$. We have

$$
\Sigma_{1}=\Sigma_{1}^{(1)}+\Sigma_{1}^{(2)},
$$

where $\Sigma_{1}^{(1)}$ and $\Sigma_{1}^{(2)}$ denote the respective contributions of the summands with $s_{1} \neq 0$ and $s_{1}=0$ on the right-hand side of (4.20). Obviously

$$
\Sigma_{1}^{(2)} \ll M L^{2} K_{0}^{2}
$$

Using the well known estimate for the linear exponential sums and (4.12), (4.14), (4.15), (4.20), (4.22) we get

$$
\begin{aligned}
& \Sigma_{1}^{(1)} \ll \sum_{k_{1}, k_{2} \leq K_{0}}\left[k_{1}, k_{2}\right] \sum_{\substack{L<l_{1}, l_{2} \leq L_{1} \\
l_{1} \neq l_{2}}} \\
& \times \sum_{0<|s| \leq 2 M /\left[k_{1}, k_{2}\right]} \min \left(\frac{M}{\left[k_{1}, k_{2}\right]}, \frac{1}{\left\|2 \alpha\left(l_{1}^{2}-l_{2}^{2}\right)\left[k_{1}, k_{2}\right]^{2} s\right\|}\right) \\
& \ll K_{0}^{3} \sum_{h \leq K_{0}^{2}} \sum_{\substack{L<l_{1}, l_{2} \leq L_{1} \\
l_{1} \neq l_{2}}} \sum_{0<|s| \leq 2 M} \min \left(M, \frac{1}{\left\|2 \alpha\left(l_{1}^{2}-l_{2}^{2}\right) h^{2} s\right\|}\right) \\
& =K_{0}^{3} \sum_{h \leq K_{0}^{2}} \sum_{t_{1}, t_{2}}\left(\sum_{\substack{L<l_{1}, l_{2} \leq L_{1} \\
l_{1}-l_{2}=t_{1}, l_{1}+l_{2}=t_{2} \\
l_{1} \neq l_{2}}} 1\right) \sum_{0<|s| \leq 2 M} \min \left(M, \frac{1}{\left\|2 \alpha t_{1} t_{2} h^{2} s\right\|}\right) \\
& \ll K_{0}^{3} \sum_{h \leq K_{0}^{2}} \sum_{\substack{0<\left|t_{1}\right| \leq L \\
1 \leq t_{2} \leq 4 L}} \sum_{0<|s| \leq 2 M} \min \left(M, \frac{1}{\left\|2 \alpha t_{1} t_{2} h^{2} s\right\|}\right)
\end{aligned}
$$




$$
\begin{aligned}
& \ll K_{0}^{3} \sum_{h \leq K_{0}^{2}} \sum_{1 \leq t_{1}, t_{2} \leq 4 L} \sum_{1 \leq s \leq 2 M} \min \left(M, \frac{1}{\left\|2 \alpha t_{1} t_{2} h^{2} s\right\|}\right) \\
& \ll K_{0}^{3} \sum_{1 \leq m \leq 64 K_{0}^{4} L^{2} M} \tau_{5}(m) \min \left(M, \frac{1}{\|\alpha m\|}\right) .
\end{aligned}
$$

To get rid of $\tau_{5}(\mathrm{~m})$ weights we apply Cauchy's inequality. Then we use Lemma 2.2 of Vaughan [27] and $(2.3),(2.4),(4.12),(4.18)$ to obtain $\Sigma_{1}^{(1)} \ll$ $M^{2} L^{2} \mathcal{L}^{-140 A}$. We leave the calculations to the reader. The last estimate and (4.23), (4.24) give

$$
\Sigma_{1} \ll M^{2} L^{2} \mathcal{L}^{-140 A} .
$$

Consider now the sum $\Sigma_{2}$. According to (4.21) we have

$$
\Sigma_{2} \ll \mathcal{L} \max _{K_{0} \leq T \leq K^{2}}\left(T \Sigma_{2}^{(1)}\right)
$$

where

$$
\begin{aligned}
\Sigma_{2}^{(1)}=\Sigma_{2}^{(1)}(T)= & \sum_{\substack{k_{1}, k_{2} \leq K \\
\left(k_{1} k_{2}, 2\right)=1 \\
T \leq\left[k_{1}, k_{2}\right] \leq 2 T}} \sum_{\substack{L<l_{1}, l_{2} \leq L_{1}, l_{1} \neq l_{2} \\
\left(k_{1}, l_{1}\right)=\left(k_{2}, l_{2}\right)=1 \\
l_{1} \equiv l_{2}\left(\left(k_{1}, k_{2}\right)\right)}} \\
& \times \sum_{\left|s_{1}\right| \leq 2 H_{1}-2 H}\left|\sum_{H^{\prime}<t \leq H_{1}^{\prime}} e\left(2 \alpha s_{1}\left[k_{1}, k_{2}\right]^{2}\left(l_{1}^{2}-l_{2}^{2}\right) t\right)\right| .
\end{aligned}
$$

The interval of summation over $t$ in the sum above depends on the other variables, which is not convenient. To get rid of this dependence, we apply Lemma 2.2 of Bombieri and Iwaniec [2] and estimate $\Sigma_{2}^{(1)}$ by means of the mean value of a similar sum, in which the interval of summation over $t$ does not depend on $k_{i}, l_{i}, s_{1}$. In the new sum we may already extend the domain of summation over $k_{i}, l_{i}, s_{1}$. After that the quantity under consideration does not decrease. More precisely, using (4.14), (4.15), (4.22) and the lemma mentioned above, we obtain

$$
\begin{aligned}
\Sigma_{2}^{(1)} \leq & \sum_{\substack{k_{1}, k_{2} \leq K \\
T \leq\left[k_{1}, k_{2}\right] \leq 2 T}} \sum_{\substack{L<l_{1}, l_{2} \leq L_{1} \\
l_{1} \neq l_{2}}} \sum_{\substack{|s| \leq 2 M / T\\
}} \times\left.\sum_{-\infty /(4 T)<t \leq 4 M / T}^{\infty} \mathcal{K}(\theta)\right|_{-\infty} ^{\infty} e(\theta t) e\left(2 \alpha s\left[k_{1}, k_{2}\right]^{2}\left(l_{1}^{2}-l_{2}^{2}\right) t\right) \mid d \theta \\
= & \int_{-\infty}^{\infty} \mathcal{K}(\theta) \Sigma_{2}^{(2)}(\theta, T) d \theta
\end{aligned}
$$

where

$$
\mathcal{K}(\theta)=\min \left(15 M /(4 T)+1,(\pi|\theta|)^{-1},(\pi \theta)^{-2}\right)
$$


and

$$
\begin{aligned}
\Sigma_{2}^{(2)}=\Sigma_{2}^{(2)}(\theta, T)= & \sum_{\substack{k_{1}, k_{2} \leq K \\
T<\left[k_{1}, k_{2}\right] \leq 2 T}} \sum_{\substack{L<l_{1}, l_{2} \leq L_{1} \\
l_{1} \neq l_{2}}} \sum_{|s| \leq 2 M / T} e\left(2 \alpha s\left[k_{1}, k_{2}\right]^{2}\left(l_{1}^{2}-l_{2}^{2}\right) t+\theta t\right) \mid . \\
& \times \sum_{\substack{M /(4 T)<t \leq 4 M / T}} e .
\end{aligned}
$$

From (4.27), (4.28) we get

$$
\Sigma_{2}^{(1)} \ll \mathcal{L} \max _{0 \leq \theta \leq 1} \Sigma_{2}^{(2)} .
$$

Consider $\Sigma_{2}^{(2)}$. We have

$$
\begin{aligned}
& \text { (4.30) } \Sigma_{2}^{(2)}=\sum_{T<h \leq 2 T}\left(\sum_{\substack{k_{1}, k_{2} \leq K \\
\left[k_{1}, k_{2}\right]=h}} 1\right) \sum_{\substack{L<l_{1}, l_{2} \leq L_{1} \\
l_{1} \neq l_{2}}} \\
& \times \sum_{|s| \leq 2 M / T}\left|\sum_{M /(4 T)<t \leq 4 M / T} e\left(2 \alpha s^{2}\left(l_{1}^{2}-l_{2}^{2}\right) t+\theta t\right)\right| \\
& \ll \sum_{T<h \leq 2 T} \tau^{2}(h) \sum_{\substack{L<l_{1}, l_{2} \leq L_{1} \\
l_{1} \neq l_{2}}} \\
& \times \sum_{|s| \leq 2 M / T}\left|\sum_{M /(4 T)<t \leq 4 M / T} e\left(2 \alpha s h^{2}\left(l_{1}^{2}-l_{2}^{2}\right) t+\theta t\right)\right| \\
& =\sum_{T<h \leq 2 T} \tau^{2}(h) \sum_{t_{1}, t_{2}}\left(\sum_{\substack{L<l_{1}, l_{2} \leq L_{1} \\
l_{1}-l_{2}=t_{1}, l_{1}+l_{2}=t_{2} \\
l_{1} \neq l_{2}}} 1\right) \\
& \times \sum_{|s| \leq 2 M / T}\left|\sum_{M /(4 T)<t \leq 4 M / T} e\left(2 \alpha \operatorname{sh}^{2} t_{1} t_{2} t+\theta t\right)\right| \\
& \ll \sum_{T<h \leq 2 T} \tau^{2}(h) \sum_{0<\left|t_{1}\right|,\left|t_{2}\right| \leq 4 L} \\
& \times \sum_{|s| \leq 2 M / T}\left|\sum_{M /(4 T)<t \leq 4 M / T} e\left(2 \alpha s^{2} t_{1} t_{2} t+\theta t\right)\right| \\
& \ll \sum_{T<h \leq 2 T} \tau^{2}(h) \sum_{0<\left|t_{1}\right|,\left|t_{2}\right| \leq 4 L} \\
& \times \sum_{0<|s| \leq 2 M / T}\left|\sum_{M /(4 T)<t \leq 4 M / T} e\left(2 \alpha \operatorname{sh}^{2} t_{1} t_{2} t+\theta t\right)\right|+M L^{2} \mathcal{L}^{3}
\end{aligned}
$$

$\ll M L^{2} \mathcal{L}^{3}+\Sigma_{2}^{(3)}$, 
where

$$
\begin{aligned}
\Sigma_{2}^{(3)}= & \sum_{T<h \leq 2 T} \tau^{2}(h) \\
& \times \sum_{0<|m| \leq 32 M L^{2} / T} \tau^{3}(|m|) \mid \sum_{M /(4 T)<t \leq 4 M / T} e\left(2 \alpha h^{2} m t+\theta t\right) .
\end{aligned}
$$

We use the Cauchy inequality to get

$$
\begin{aligned}
\left(\Sigma_{2}^{(3)}\right)^{2} & \leq\left(\sum_{T<h \leq 2 T} \tau^{4}(h) \sum_{0<|m| \leq 32 M L^{2} / T} \tau^{6}(|m|)\right) \Sigma_{2}^{(4)} \\
& \ll M L^{2} \mathcal{L}^{100} \Sigma_{2}^{(4)},
\end{aligned}
$$

where

$$
\Sigma_{2}^{(4)}=\sum_{T<h \leq 2 T} \sum_{0<|m| \leq 32 M L^{2} / T}\left|\sum_{M /(4 T)<t \leq 4 M / T} e\left(2 \alpha h^{2} m t+\theta t\right)\right|^{2} .
$$

For the last sum we have

$$
\begin{aligned}
\Sigma_{2}^{(4)}= & \sum_{T<h \leq 2 T} \sum_{0<|m| \leq 32 M L^{2} / T} e\left(\left(2 \alpha m h^{2}+\theta\right)\left(t_{1}-t_{2}\right)\right) \\
& \times \sum_{M /(4 T)<t_{1}, t_{2} \leq 4 M / T} \sum_{0<|m| \leq 32 M L^{2} / T \quad M /(4 T)<t_{1}, t_{2} \leq 4 M / T} e\left(\left(2 \alpha m h^{2}\right)\left(t_{1}-t_{2}\right)\right) \mid \\
& \times\left|\sum_{T<h \leq 2 T} \sum^{2} \sum^{2} \sum_{0<|m| \leq 32 M L^{2} / T \quad} \sum_{0<|l| \leq 4 M / T}\right| \sum_{T<h \leq 2 T} e\left(2 \alpha m h^{2} l\right) \mid \\
\ll & \frac{M^{2} L^{2}}{T}+\frac{M}{T} \Sigma_{2}^{(5)},
\end{aligned}
$$

where

$$
\Sigma_{2}^{(5)}=\sum_{0<|s| \leq 256 M^{2} L^{2} / T^{2}} \tau(|s|)\left|\sum_{T<h \leq 2 T} e\left(\alpha s h^{2}\right)\right| .
$$

By Cauchy's inequality we obtain

$$
\begin{aligned}
\left(\Sigma_{2}^{(5)}\right)^{2} \ll & \left(\sum_{1 \leq s \leq 256 M^{2} L^{2} / T^{2}} \tau^{2}(s)\right) \\
& \times\left(\sum_{1 \leq s \leq 256 M^{2} L^{2} / T^{2}}\left|\sum_{T<h \leq 2 T} e\left(\alpha s h^{2}\right)\right|^{2}\right)
\end{aligned}
$$




$$
\begin{aligned}
& \ll \frac{M^{2} L^{2}}{T^{2}} \mathcal{L}^{3} \sum_{1 \leq s \leq 256 M^{2} L^{2} / T^{2}} \sum_{T<h_{1}, h_{2} \leq 2 T} e\left(\alpha s\left(h_{1}^{2}-h_{2}^{2}\right)\right) \\
& \ll \frac{M^{4} L^{4}}{T^{3}} \mathcal{L}^{3}+\frac{M^{2} L^{2}}{T^{2}} \mathcal{L}^{3}\left|\Sigma_{2}^{(6)}\right|,
\end{aligned}
$$

where

$$
\Sigma_{2}^{(6)}=\sum_{1 \leq s \leq 256 M^{2} L^{2} / T^{2}} \sum_{\substack{T<h_{1}, h_{2} \leq 2 T \\ h_{1} \neq h_{2}}} e\left(\alpha s\left(h_{1}^{2}-h_{2}^{2}\right)\right) .
$$

Applying the estimate for the linear sums again we get

$$
\begin{aligned}
\left|\Sigma_{2}^{(6)}\right| & =\left|\sum_{m_{1}, m_{2}}\left(\sum_{\substack{T<h_{1}, h_{2} \leq 2 T \\
h_{1}-h_{2}=m_{1}, h_{1}+h_{2}=m_{2} \\
h_{1} \neq h_{2}}} 1\right) \sum_{1 \leq s \leq 256 M^{2} L^{2} / T^{2}} e\left(\alpha s m_{1} m_{2}\right)\right| \\
& \ll \sum_{0<\left|m_{1}\right|,\left|m_{2}\right| \leq 4 T} \mid \sum_{\substack{1 \leq s \leq 256 M^{2} L^{2} / T^{2} \\
1 \leq m_{1}, m_{2} \leq 4 T}} \min \left(\frac{M^{2} L^{2}}{T^{2}}, \frac{1}{\left\|\alpha m_{1} m_{2}\right\|}\right) \\
& \ll \sum_{1 \leq m \leq 16 T^{2}} \tau(m) \min \left(\frac{M^{2} L^{2}}{T^{2}}, \frac{1}{\|\alpha m\|}\right) .
\end{aligned}
$$

Now we proceed as in the estimation of $\Sigma_{1}^{(1)}$ to get

$$
\Sigma_{2}^{(6)} \ll M^{2} L^{2} \mathcal{L}^{2-50 A} .
$$

The inequalities (2.17), (4.12), (4.18), (4.26), (4.29)-(4.34) imply

$$
\Sigma_{2} \ll M^{2} L^{2} \mathcal{L}^{-12 A} \text {. }
$$

Taking into account (4.12), (4.16), (4.19), (4.25) and (4.35), we find that

$$
\left|W_{2}\right| \ll X \mathcal{L}^{50-3 A} \text {. }
$$

Let us now estimate type I sums. Consider, for example, the sum $W_{1}$. According to (2.18) and (4.11) we have

$$
\left|W_{1}\right| \ll \mathcal{L}^{2} \max _{1 / 2 \leq T \leq K} \Sigma_{3}
$$

where

$$
\Sigma_{3}=\Sigma_{3}(T)=\sum_{\substack{T<k \leq 2 T \\(k, 2)=1}} \tau(k) \sum_{\substack{M<m \leq M_{1} \\(m, k)=1}} \tau_{5}(m)\left|\sum_{\substack{L^{\prime}<l \leq L_{1}^{\prime} \\ m l+2 \equiv 0(k)}} e\left(\alpha m^{2} l^{2}\right)\right|,
$$

and

$$
L^{\prime}=\max (L, Y / m), \quad L_{1}^{\prime}=\min \left(L_{1}, 2 Y / m\right)
$$


For any $m$ coprime to $k$ we define $\bar{m}$ by $m \bar{m} \equiv 1(k), 0 \leq \bar{m}<k$. Let

$$
R=\left(L^{\prime}+2 \bar{m}\right) / k, \quad R_{1}=\left(L_{1}^{\prime}+2 \bar{m}\right) / k .
$$

By Cauchy's inequality we get

$$
\begin{aligned}
\left(\Sigma_{3}\right)^{2} \ll & \left(\sum_{T<k \leq 2 T} \tau^{2}(k) \sum_{M<m \leq M_{1}} \tau_{5}^{2}(m)\right) \\
& \times\left(\sum_{\substack{T<k \leq 2 T \\
(k, 2)=1}} \sum_{\substack{M<m \leq M_{1} \\
(m, k)=1}}\left|\sum_{R<r \leq R_{1}} e\left(\alpha m^{2}(-2 \bar{m}+r k)^{2}\right)\right|^{2}\right) \\
\ll & M T \mathcal{L}^{100} \sum_{\substack{T<k \leq 2 T \\
(k, 2)=1}} \sum_{\substack{M<m \leq M_{1} \\
(m, k)=1}}\left|\sum_{R<r \leq R_{1}} e\left(\alpha m^{2}\left(r^{2} k^{2}-4 \bar{m} r k\right)\right)\right|^{2} \\
= & M T \mathcal{L}^{100} \sum_{\substack{T<k \leq 2 T \\
(k, 2)=1}} \sum_{\substack{M<m \leq M_{1} \\
(m, k)=1}} e\left(\alpha m^{2}\left(k^{2}\left(r_{1}^{2}-r_{2}^{2}\right)-4 \bar{m} k\left(r_{1}-r_{2}\right)\right)\right) \\
& \times \sum_{R<r_{1}, r_{2} \leq R_{1}} M^{2} L T \mathcal{L}^{100}+M T \mathcal{L}^{100}\left|\Sigma_{3}^{(1)}\right|,
\end{aligned}
$$

where

$$
\begin{aligned}
\Sigma_{3}^{(1)}= & \sum_{\substack{T<k \leq 2 T \\
(k, 2)=1}} \sum_{\substack{M<m \leq M_{1} \\
(m, k)=1}} e\left(\alpha m^{2}\left(k^{2}\left(r_{1}^{2}-r_{2}^{2}\right)-4 \bar{m} k\left(r_{1}-r_{2}\right)\right)\right) . \\
& \times \sum_{\substack{R<r_{1}, r_{2} \leq R_{1} \\
r_{1} \neq r_{2}}}
\end{aligned}
$$

We have

$$
\begin{aligned}
\left|\Sigma_{3}^{(1)}\right|= & \left|\sum_{\substack{T<k<2 T \\
(k, 2)=1}} \sum_{\substack{M<m \leq M_{1} \\
(m, k)=1}} e\left(\alpha m^{2}\left(k^{2} s_{1} s_{2}-4 \bar{m} k s_{1}\right)\right) \sum_{\substack{s_{1}, s_{2} \\
s_{1} \neq 0 \\
R<r_{1}, r_{2} \leq R_{1} \\
r_{1}-r_{2}=s_{1} \\
r_{1}+r_{2}=s_{2}}} 1\right| \\
= & \left|\sum_{\substack{T<k<2 T \\
(k, 2)=1}} \sum_{\substack{M<m \leq M_{1} \\
(m, k)=1}} e\left(\alpha m^{2}\left(k^{2} s_{1} s_{2}-4 \bar{m} k s_{1}\right)\right)\right| \\
& \times \sum_{\substack{s_{1}, s_{2}: s_{1} \neq 0 \\
2 R<s_{1}+s_{2}, s_{2}-s_{1} \leq 2 R_{1} \\
s_{1} \equiv s_{2}(2)}}
\end{aligned}
$$




$$
\begin{aligned}
& \ll \sum_{\substack{T<k \leq 2 T \\
(k, 2)=1}} \sum_{\substack{M<m \leq M_{1} \\
(m, k)=1}} \sum_{0<\left|s_{1}\right| \leq 10 L / T}\left|\sum_{\substack{s_{2}: s_{2} \equiv s_{1}(2) \\
2 R-s_{1}<s_{2} \leq 2 R_{1}-s_{1} \\
2 R+s_{1} \leq s_{2} \leq 2 R_{1}+s_{1}}} e\left(\alpha m^{2} k^{2} s_{1} s_{2}\right)\right| \\
& \ll \sum_{\substack{T<k \leq 2 T \\
k, 2)=1}} \sum_{\substack{M<m \leq M_{1} \\
(m, k)=1}} \sum_{1 \leq s_{1} \leq 10 L / T}\left|\sum_{\begin{array}{c}
R<t \leq R_{1} \\
R-s_{1}<t \leq R_{1}-s_{1}
\end{array}} e\left(2 \alpha m^{2} k^{2} s_{1} t\right)\right| .
\end{aligned}
$$

First we consider the case

$$
M T \leq K_{0},
$$

where $K_{0}$ is defined by (4.18). We apply Cauchy's inequality, Lemma 2.2 of Vaughan [27] and also (2.3), (2.4), (4.11), (4.18), (4.42) to get

$$
\begin{aligned}
\left|\Sigma_{3}^{(1)}\right| & \ll \sum_{T<k \leq 2 T} \sum_{M<m \leq M_{1}} \sum_{1 \leq s \leq 20 L} \min \left(L, \frac{1}{\left\|2 \alpha m^{2} k^{2} s\right\|}\right) \\
& \ll \sum_{1 \leq n \leq 640 K_{0}^{2} L} \tau^{3}(n) \min \left(L, \frac{1}{\|\alpha n\|}\right) \ll X^{2} \mathcal{L}^{-300 A} .
\end{aligned}
$$

Hence, by (4.11), (4.40), (4.42), (4.43) we find that

$$
\Sigma_{3} \ll X \mathcal{L}^{-100 A} \quad \text { if } M T \leq K_{0} .
$$

Consider now the case

$$
M T>K_{0} .
$$

Using (4.38), (4.39), (4.41) and Lemma 2.2 of Bombieri and Iwaniec [2] we obtain

$$
\left|\Sigma_{3}^{(1)}\right| \ll \mathcal{L} \max _{0 \leq \theta \leq 1} \Sigma_{3}^{(2)},
$$

where

$$
\begin{aligned}
\Sigma_{3}^{(2)} & =\Sigma_{3}^{(2)}(\theta, T) \\
& =\sum_{T<k \leq 2 T} \sum_{M<m \leq M_{1}} \sum_{1 \leq s \leq 10 L / T}\left|\sum_{L /(4 T)<t \leq 4 L / T} e\left(2 \alpha k^{2} m^{2} s t+\theta t\right)\right| .
\end{aligned}
$$

It is clear that

$$
\Sigma_{3}^{(2)} \ll \sum_{M T<h \leq 4 M T} \tau(h) \sum_{1 \leq s \leq 10 L / T}\left|\sum_{L /(4 T)<t \leq 4 L / T} e\left(2 \alpha h^{2} s t+\theta t\right)\right| .
$$

Hence an application of Cauchy's inequality gives 


$$
\begin{aligned}
\left(\Sigma_{3}^{(2)}\right)^{2} & \ll\left(\sum_{M T<h \leq 4 M T} \tau^{2}(h) \sum_{1 \leq s \leq 10 L / T} 1\right) \\
& \times\left(\sum_{M T<h \leq 4 M T} \sum_{1 \leq s \leq 10 L / T}\left|\sum_{L /(4 T)<t \leq 4 L / T} e\left(2 \alpha h^{2} s t+\theta t\right)\right|^{2}\right)
\end{aligned}
$$

$$
\ll M L \mathcal{L}^{3} \sum_{M T<h \leq 4 M T} \sum_{1 \leq s \leq 10 L / T} \sum_{L /(4 T)<t_{1}, t_{2} \leq 4 L / T} e\left(\left(2 \alpha h^{2} s+\theta\right)\left(t_{1}-t_{2}\right)\right)
$$$$
\ll M L \mathcal{L}^{3} \sum_{1 \leq s \leq 10 L / T} \sum_{L /(4 T)<t_{1}, t_{2} \leq 4 L / T}\left|\sum_{M T<h \leq 4 M T} e\left(2 \alpha h^{2} s\left(t_{1}-t_{2}\right)\right)\right|
$$$$
\ll \frac{M^{2} L^{3}}{T} \mathcal{L}^{3}+M L \mathcal{L}^{3} \Sigma_{3}^{(3)}
$$

where

$$
\Sigma_{3}^{(3)}=\sum_{1 \leq s \leq 10 L / T} \sum_{\substack{L /(4 T)<t_{1}, t_{2} \leq 4 L / T \\ t_{1} \neq t_{2}}}\left|\sum_{M T<h \leq 4 M T} e\left(2 \alpha h^{2} s\left(t_{1}-t_{2}\right)\right)\right| .
$$

For the last sum we have

$$
\begin{aligned}
\Sigma_{3}^{(3)} & =\sum_{1 \leq s \leq 10 L / T} \sum_{\substack{0<|t| \leq 4 L / T \\
1 \leq u \leq 8 L / T}}\left(\sum_{\substack{L /(4 T)<t_{1}, t_{2} \leq 4 L / T \\
t_{1}-t_{2}=t \\
t_{1}+t_{2}=u}} 1\right)\left|\sum_{M T<h \leq 4 M T} e\left(2 \alpha h^{2} s t\right)\right| \\
& \ll \frac{L}{T} \sum_{1 \leq s \leq 10 L / T} \sum_{1 \leq t \leq 4 L / T}\left|\sum_{M T<h \leq 4 M T} e\left(2 \alpha h^{2} s t\right)\right| \\
& \ll \frac{L}{T} \sum_{1 \leq m \leq 80 L^{2} / T^{2}} \tau(m)\left|\sum_{M T<h \leq 4 M T} e\left(\alpha h^{2} m\right)\right| .
\end{aligned}
$$

Hence

$$
\begin{aligned}
\left(\Sigma_{3}^{(3)}\right)^{2} \ll & \frac{L^{2}}{T^{2}}\left(\sum_{1 \leq m \leq 80 L^{2} / T^{2}} \tau^{2}(m)\right) \\
& \times\left(\sum_{1 \leq m \leq 80 L^{2} / T^{2}}\left|\sum_{M T<h \leq 4 M T} e\left(\alpha h^{2} m\right)\right|^{2}\right) \\
\ll & \frac{L^{4}}{T^{4}} \mathcal{L}^{3} \sum_{1 \leq m \leq 80 L^{2} / T^{2} \quad} \sum_{M T<h_{1}, h_{2} \leq 4 M T} e\left(\alpha\left(h_{1}^{2}-h_{2}^{2}\right) m\right) \\
\ll & \frac{L^{6} M}{T^{5}} \mathcal{L}^{3}+\frac{L^{4}}{T^{4}} \mathcal{L}^{3} \Sigma_{3}^{(4)},
\end{aligned}
$$


where

$$
\Sigma_{3}^{(4)}=\sum_{\substack{M T<h_{1}, h_{2} \leq 4 M T \\ h_{1} \neq h_{2}}}\left|\sum_{1 \leq m \leq 80 L^{2} / T^{2}} e\left(\alpha\left(h_{1}^{2}-h_{2}^{2}\right) m\right)\right| .
$$

So we get as before

$$
\begin{aligned}
(4.49) \quad \Sigma_{3}^{(4)} & =\sum_{0<\left|s_{1}\right|,\left|s_{2}\right| \leq 8 M T}\left(\sum_{\begin{array}{c}
T<h_{1}, h_{2} \leq 4 M T \\
h_{1}-h_{2}=s_{1} \\
h_{1}+h_{2}=s_{2}
\end{array}} 1\right) \\
& \times\left|\sum_{1 \leq m \leq 80 L^{2} / T^{2}} e\left(\alpha s_{1} s_{2} m\right)\right| \\
& \ll \sum_{1 \leq s_{1}, s_{2} \leq 8 M T}\left|\sum_{1 \leq m \leq 80 L^{2} / T^{2}} e\left(\alpha s_{1} s_{2} m\right)\right| \\
& \ll \sum_{1 \leq s_{1}, s_{2} \leq 8 M T} \min \left(\frac{L^{2}}{T^{2}}, \frac{1}{\left\|\alpha s_{1} s_{2}\right\|}\right) \\
& \ll \sum_{1 \leq s \leq 64 M^{2} T^{2}} \tau(s) \min \left(\frac{L^{2}}{T^{2}}, \frac{1}{\|\alpha s\|}\right) \ll M^{2} L^{2} \mathcal{L}^{2-50 A} .
\end{aligned}
$$

Using (2.17), (4.11), (4.18), (4.40), (4.45)-(4.49) we find that

$$
\Sigma_{3} \ll X \mathcal{L}^{-6 A} \quad \text { if } M T>K_{0} .
$$

Hence by $(4.37),(4.44)$ and $(4.50)$ we obtain the estimate

$$
\left|W_{1}\right| \ll X \mathcal{L}^{2-6 A} \text {. }
$$

We treat type I sums $W_{1}^{\prime}$ in the same way and we find that

$$
\left|W_{1}^{\prime}\right| \ll X \mathcal{L}^{2-6 A} \text {. }
$$

The estimate (4.4) follows from (4.10), (4.36), (4.51) and (4.52). Now the proof of the estimate $(2.23)$ for $\mathcal{U}_{2}$ is complete.

5. Proof of the Proposition-major arcs. In this section we prove that for the sum $\mathcal{U}_{1}$, defined by (2.20), the estimate (2.23) holds. However, now we do not need such a restrictive upper bound for $K_{3}$, as in Section 4 . Now we assume that

$$
K_{i} \leq X^{1 / 2} \mathcal{L}^{-20000 A}, \quad i=1,2,3 .
$$

According to (2.4) and (2.6) we have

$$
I_{1}=\sum_{q<Q} \sum_{\substack{0 \leq a \leq q-1 \\(a, q)=1}} H(a, q),
$$


where

$$
\begin{aligned}
H(a, q)= & \int_{-1 /(q \tau)}^{1 /(q \tau)} S_{k_{1}}\left(\frac{a}{q}+\alpha\right) \\
& S_{k_{2}}\left(\frac{a}{q}+\alpha\right) \\
& \times S_{k_{3}}\left(\frac{a}{q}+\alpha\right) e\left(-n\left(\frac{a}{q}+\alpha\right)\right) d \alpha
\end{aligned}
$$

and where $S_{k}(\alpha)$ is defined by (2.2). Denote

$$
M(\alpha)=\sum_{m \leq N} \frac{1}{2 \sqrt{m}} e(\alpha m), \quad \Delta(y, h)=\max _{z \leq y} \max _{(l, h)=1}\left|\sum_{\substack{p \leq z \\ p \equiv l(h)}} \log p-\frac{z}{\varphi(h)}\right|
$$

and let $s_{k}(a, q)$ be defined by $(2.7)$. We write

$$
S_{k}\left(\frac{a}{q}+\alpha\right)=\frac{s_{k}(a, q)}{\varphi(k)} M(\alpha)+\mathcal{G}(\alpha ; k, q, a) .
$$

For $\alpha, a, q$ satisfying

$$
|\alpha| \leq(q \tau)^{-1}, \quad 0 \leq a<q<Q, \quad(a, q)=1
$$

and for $k \leq X^{1 / 2} \mathcal{L}^{-20000 A}$ we have

$$
\mathcal{G}(\alpha ; k, q, a) \ll(1+\Delta(X,[k, q])) \frac{X^{2}}{\tau} .
$$

The calculations are similar to those in Section 4.1 of [21], so we do not present them here. We define

$$
\Gamma_{i}(\alpha, q, a)=\sum_{k \leq K_{i}} \beta_{i}(k) \mathcal{G}(\alpha ; k, q, a), \quad i=1,2,3 .
$$

By (5.6) we get

$$
\begin{aligned}
\max _{\substack{\alpha, q, a \\
(5.5)}}\left|\Gamma_{i}(\alpha, q, a)\right| & \ll \frac{X^{2}}{\tau} \sum_{q \leq Q} \sum_{k \leq K_{i}} \tau(k)(1+\Delta(X,[k, q])) \\
& \ll \frac{X^{2}}{\tau} \sum_{h \leq K_{i} Q} \tau^{3}(h)(1+\Delta(X, h)) .
\end{aligned}
$$

Applying Cauchy's inequality and Bombieri-Vinogradov's theorem (Chapter 28 of Davenport [4]) and using (2.3), (5.1) we get

$$
\max _{\substack{\alpha, q, a \\(5.5)}}\left|\Gamma_{i}(\alpha, q, a)\right| \ll X \mathcal{L}^{-7000 A}, \quad i=1,2,3 .
$$

Define

$$
\mathcal{S}_{i}=S_{k_{i}}\left(\frac{a}{q}+\alpha\right), \quad \mathcal{M}_{i}=\frac{s_{k_{i}}(a, q)}{\varphi\left(k_{i}\right)} M(\alpha), \quad \mathcal{G}_{i}=\mathcal{S}_{i}-\mathcal{M}_{i}
$$


We use (5.2)-(5.4), (5.9) and the identity

$$
\mathcal{S}_{1} \mathcal{S}_{2} \mathcal{S}_{3}=\mathcal{M}_{1} \mathcal{M}_{2} \mathcal{M}_{3}+\mathcal{S}_{1} \mathcal{S}_{2} \mathcal{G}_{3}+\mathcal{S}_{1} \mathcal{G}_{2} \mathcal{M}_{3}+\mathcal{G}_{1} \mathcal{M}_{2} \mathcal{M}_{3}
$$

to get

$$
I_{1}=J^{\prime}+J_{1}+J_{2}+J_{3},
$$

where

$$
J^{\prime}=\sum_{q<Q} \sum_{\substack{0 \leq a \leq q-1 \\(a, q)=1}} \int_{-1 /(q \tau)}^{1 /(q \tau)} \mathcal{M}_{1} \mathcal{M}_{2} \mathcal{M}_{3} e\left(-n\left(\frac{a}{q}+\alpha\right)\right) d \alpha
$$

and where $J_{1}, J_{2}$ and $J_{3}$ are the contributions of the other summands.

Consequently,

$$
\mathcal{U}_{1} \ll \mathcal{U}^{\prime}+\mathcal{Z}_{1}+\mathcal{Z}_{2}+\mathcal{Z}_{3},
$$

where

$$
\begin{gathered}
\mathcal{U}^{\prime}=\sum_{n \leq N}^{*}\left|\sum_{\substack{k_{i} \leq K_{i} \\
i=1,2,3}} \beta_{1}\left(k_{1}\right) \beta_{2}\left(k_{2}\right) \beta_{3}\left(k_{3}\right)\left(J^{\prime}-\frac{\pi}{4} \sqrt{n} \frac{\mathfrak{S}\left(n ; Q ; k_{1}, k_{2}, k_{3}\right)}{\varphi\left(k_{1}\right) \varphi\left(k_{2}\right) \varphi\left(k_{3}\right)}\right)\right|, \\
\mathcal{Z}_{1}=\sum_{n \leq N} \mid \sum_{\substack{k_{i} \leq K_{i} \\
i=1,2,3}} \beta_{1}\left(k_{1}\right) \beta_{2}\left(k_{2}\right) \beta_{3}\left(k_{3}\right) \\
\quad \times \sum_{q<Q} \sum_{\substack{0 \leq a \leq q-1 \\
(a, q)=1}} \int_{-1 /(q \tau)}^{1 /(q \tau)} \mathcal{S}_{1} \mathcal{S}_{2} \mathcal{G}_{3} e\left(-n\left(\frac{a}{q}+\alpha\right)\right) d \alpha \mid,
\end{gathered}
$$

the definitions of $\mathcal{Z}_{2}$ and $\mathcal{Z}_{3}$ are clear. First we show that

$$
\mathcal{Z}_{i} \ll X^{3} \mathcal{L}^{-A}, \quad i=1,2,3 .
$$

Consider, for example, $\mathcal{Z}_{1}$. We have

$$
\begin{aligned}
\mathcal{Z}_{1} \ll \sum_{q<Q} \sum_{\substack{0 \leq a \leq q-1 \\
(a, q)=1}} \sum_{n \leq N} \mid \int_{-1 /(q \tau)}^{1 /(q \tau)} \mathcal{K}_{1}\left(\frac{a}{q}+\alpha\right) \\
\quad \times \mathcal{K}_{2}\left(\frac{a}{q}+\alpha\right) \Gamma_{3}(\alpha, q, a) e\left(-n\left(\frac{a}{q}+\alpha\right)\right) d \alpha \mid,
\end{aligned}
$$

where $\mathcal{K}_{i}(\alpha)$ are defined by (4.1) and $\Gamma_{3}(\alpha, q, a)$ by (5.7). We apply the Cauchy and Bessel inequalities to get 


$$
\begin{aligned}
& \left.\mathcal{Z}_{1}^{2} \ll Q^{2} X^{2} \sum_{q<Q} \sum_{\substack{0 \leq a \leq q-1 \\
(a, q)=1}} \sum_{n \leq N}\right|_{-1 /(q \tau)} ^{1 /(q \tau)} \int_{\substack{q \\
(1 / 2)}} \mathcal{K}_{1}\left(\frac{a}{q}+\alpha\right) \\
& \times\left.\mathcal{K}_{2}\left(\frac{a}{q}+\alpha\right) \Gamma_{3}(\alpha, q, a) e\left(-n\left(\frac{a}{q}+\alpha\right)\right) d \alpha\right|^{2} \\
& \ll Q^{2} X^{2} \sum_{q<Q} \sum_{\substack{0 \leq a \leq q-1 \\
(a, q)=1}}^{1 /(q \tau)} \int_{-1 /(q \tau)}\left|\mathcal{K}_{1}\left(\frac{a}{q}+\alpha\right) \mathcal{K}_{2}\left(\frac{a}{q}+\alpha\right) \Gamma_{3}(\alpha, q, a)\right|^{2} d \alpha \\
& \ll Q^{2} X^{2} \max _{\substack{\alpha, q, a \\
(5.5)}}\left|\Gamma_{3}(\alpha, q, a)\right|^{2} \int_{0}^{1}\left|\mathcal{K}_{1}(\alpha) \mathcal{K}_{2}(\alpha)\right|^{2} d \alpha \\
& \ll Q^{2} X^{2} \max _{\substack{\alpha, q, a \\
(5.5)}}\left|\Gamma_{3}(\alpha, q, a)\right|^{2} \int_{0}^{1}\left(\left|\mathcal{K}_{1}(\alpha)\right|^{4}+\left|\mathcal{K}_{2}(\alpha)\right|^{4}\right) d \alpha .
\end{aligned}
$$

We use $(4.3),(5.8)$ and the estimate (5.13) for $\mathcal{Z}_{1}$ follows. To treat $\mathcal{Z}_{2}$ and $\mathcal{Z}_{3}$ we also need the inequality

$$
\int_{0}^{1}\left|\sum_{k \leq K_{i}} \beta_{i}(k) \mathcal{M}_{i}\right|^{4} d \alpha \ll X^{2} \mathcal{L}^{10} \tau^{4}(q)
$$

whose proof is easy. We leave it to the reader to verify that the estimate (5.13) holds also for $\mathcal{Z}_{2}$ and $\mathcal{Z}_{3}$.

Consider the quantity $J^{\prime}$ defined by (5.11). Using (2.8) and (5.9) we get

$$
J^{\prime}=\frac{1}{\varphi\left(k_{1}\right) \varphi\left(k_{2}\right) \varphi\left(k_{3}\right)} \sum_{q<Q} t(q) \int_{-1 /(q \tau)}^{1 /(q \tau)} M^{3}(\alpha) e(-n \alpha) d \alpha .
$$

It follows from (2.9)-(2.14) that for squarefree odd integers $k_{1}, k_{2}, k_{3}$ we have

$$
t(q) \ll \tau^{3}(q) q^{-1}\left(k_{1}, q\right)\left(k_{2}, q\right)\left(k_{3}, q\right) .
$$

We also apply the well known formula

$$
\int_{-1 /(q \tau)}^{1 /(q \tau)} M^{3}(\alpha) e(-n \alpha) d \alpha=\frac{\pi}{4} \sqrt{n}+\mathcal{O}\left((q \tau)^{1 / 2}\right),
$$

whose proof is available in Vaughan [27], Chapter 2, for example.

We use $(2.3),(5.14)$ to estimate the contribution to $\mathcal{U}^{\prime}$ arising from the error term in (5.15). We leave this computation to the reader. We find

$$
\mathcal{U}^{\prime} \ll \mathcal{U}^{\prime \prime}+X^{2} \mathcal{L}^{-A}
$$


where

$\mathcal{U}^{\prime \prime}=\sum_{n \leq N}^{*} \sqrt{n}\left|\sum_{\substack{k_{i} \leq K_{i} \\ i=1,2,3}} \frac{\beta_{1}\left(k_{1}\right) \beta_{2}\left(k_{2}\right) \beta_{3}\left(k_{3}\right)}{\varphi\left(k_{1}\right) \varphi\left(k_{2}\right) \varphi\left(k_{3}\right)}\left(\sum_{q<Q} t(q)-\mathfrak{S}\left(n ; Q ; k_{1}, k_{2}, k_{3}\right)\right)\right|$.

To estimate $\mathcal{U}^{\prime \prime}$ we apply some arguments of Mikawa [16]. Consider the function

$$
\Psi(k)= \begin{cases}0 & \text { if } k \text { has a prime divisor } \geq Q, \\ 1 & \text { otherwise. }\end{cases}
$$

Let

$$
M=X^{2} Q^{-1} \quad \text { and } \quad T=8 \prod_{p<Q} p .
$$

By the definition (2.15) of $\mathfrak{S}$ we get

$$
\mathfrak{S}-\sum_{q<Q} t(q)=\sum_{Q \leq q \leq M} t(q) \Psi(q)+\sum_{M<q \leq T} t(q) \Psi(q) .
$$

Therefore

$$
\mathcal{U}^{\prime \prime} \ll \mathcal{U}^{*}+\mathcal{U}^{* *},
$$

where

$$
\begin{gathered}
\mathcal{U}^{*}=X \sum_{n \leq N}^{*} \sum_{\substack{k_{i} \leq K_{i} \\
i=1,2,3}}^{\prime} \frac{\tau\left(k_{1}\right) \tau\left(k_{2}\right) \tau\left(k_{3}\right)}{\varphi\left(k_{1}\right) \varphi\left(k_{2}\right) \varphi\left(k_{3}\right)}\left|\sum_{Q \leq q \leq M} t(q) \Psi(q)\right|, \\
\mathcal{U}^{* *}=X \sum_{n \leq N}^{*} \sum_{\substack{k_{i} \leq K_{i} \\
i=1,2,3}}^{\prime} \frac{\tau\left(k_{1}\right) \tau\left(k_{2}\right) \tau\left(k_{3}\right)}{\varphi\left(k_{1}\right) \varphi\left(k_{2}\right) \varphi\left(k_{3}\right)} \sum_{M<q \leq T}|t(q)| \Psi(q) .
\end{gathered}
$$

Here and later $\sum_{k \leq K_{i}}^{\prime}$ means that we sum over squarefree odd integers $k$ only.

Consider $\mathcal{U}^{*}$. Using Cauchy's inequality we get

$$
\begin{aligned}
\mathcal{U}^{* 2} & \ll X^{4} \mathcal{L}^{14} \sum_{\substack{k_{i} \leq K_{i} \\
i=1,2,3}}^{\prime} \frac{1}{k_{1} k_{2} k_{3}} \sum_{n \leq N}\left|\sum_{Q \leq q \leq M} t(q) \Psi(q)\right|^{2} \\
& =X^{4} \mathcal{L}^{14} \sum_{\substack{k_{i} \leq K_{i} \\
i=1,2,3}}^{\prime} \frac{1}{k_{1} k_{2} k_{3}} \mathfrak{F},
\end{aligned}
$$

say. We use the definition $(2.8)$ of $t(q)$ to represent the sum $\mathfrak{F}$ as

$$
\mathfrak{F}=\sum_{n \leq N}\left|\sum_{r \in \mathfrak{X}} \eta(r) e(-n r)\right|^{2}
$$

where

$$
\mathfrak{X}=\{a / q: Q \leq q \leq M, 1 \leq a \leq q-1,(a, q)=1\}
$$


and

$$
\eta(a / q)=s_{k_{1}}(a, q) s_{k_{2}}(a, q) s_{k_{3}}(a, q) \Psi(q) .
$$

For any $r \in \mathfrak{X}$ we set $\delta_{r}=\min \left\{\left\|r-r^{\prime}\right\|: r^{\prime} \in \mathfrak{X}, r^{\prime} \neq r\right\}$, so if $r=a / q$ then $\delta_{r} \geq(q M)^{-1}$. We apply the dual form of the large sieve inequality (see Montgomery [17], Montgomery-Vaughan [18]) to get

$$
\mathfrak{F} \ll \sum_{r \in \mathfrak{X}}\left(N+\delta_{r}^{-1}\right)|\eta(r)|^{2} \ll \sum_{Q \leq q \leq M}(N+q M) \Psi(q) \varpi(q),
$$

where

$$
\varpi(q)=\varpi\left(q ; k_{1}, k_{2}, k_{3}\right)=\sum_{\substack{0 \leq a \leq q-1 \\(a, q)=1}}\left|s_{k_{1}}(a, q) s_{k_{2}}(a, q) s_{k_{3}}(a, q)\right|^{2} .
$$

This function is multiplicative with respect to $q$ and we may easily compute $\varpi\left(p^{l}\right)$ for prime $p$. So we establish that if $k_{1}, k_{2}, k_{3}$ are squarefree odd integers then $\varpi(q) \ll q^{-2} \tau^{6}(q)\left(k_{1}, q\right)\left(k_{2}, q\right)\left(k_{3}, q\right)$. Now we use $(5.17),(5.19),(5.20)$ and after some straightforward calculations we get

$$
\mathcal{U}^{*} \ll X^{2} \mathcal{L}^{-A} \text {. }
$$

Consider $\mathcal{U}^{* *}$. We apply the estimate (5.14) to get

$$
\mathcal{U}^{* *} \ll X^{3} \mathcal{L} \sum_{M<q \leq T} \Psi(q) \frac{\tau^{3}(q)}{q}\left(\sum_{k \leq X} \frac{\tau(k)(k, q)}{k}\right)^{3} \ll X^{3} \mathcal{L}^{7} \mathfrak{T},
$$

where

$$
\begin{aligned}
\mathfrak{T} & =\sum_{M<q \leq T} \Psi(q) \frac{\tau^{9}(q)}{q} \ll \sum_{\substack{M<q_{1} \ldots q_{9} \leq T \\
q_{1} \ldots q_{9}}} \frac{\Psi\left(q_{1} \ldots q_{9}\right)}{\Psi\left(q_{1}\right) \ldots \Psi\left(q_{9}\right)} \\
& \ll \sum_{\substack{M<q_{1} \ldots q_{9} \leq T \\
q_{1} \leq q_{2} \leq \ldots \leq q_{9}}} \frac{\Psi q_{9}}{q_{1} \ldots} \\
& \ll\left(\sum_{q \leq T} \frac{\Psi(q)}{q}\right)^{8} \sum_{M^{1 / 9}<q \leq T} \frac{\Psi(q)}{q} .
\end{aligned}
$$

As in Mikawa's paper [16] we find that

$$
\sum_{M^{1 / 9}<q \leq T} \Psi(q) / q \ll \exp (-\sqrt{\mathcal{L}})
$$

and obviously $\sum_{q \leq T} \Psi(q) / q \ll \mathcal{L}$. Hence using (5.22), (5.23) we get

$$
\mathcal{U}^{* *} \ll X^{3} \mathcal{L}^{-A}
$$

The estimate $(2.23)$ for $\mathcal{U}_{1}$ is a consequence of $(5.12),(5.13),(5.16),(5.18)$, (5.21) and (5.24).

Now the proof of Theorem 1 is complete. 


\section{References}

[1] J. Brüdern and E. Fouvry, Lagrange's Four Squares Theorem with almost prime variables, J. Reine Angew. Math. 454 (1994), 59-96.

[2] E. Bombieri and H. Iwaniec, On the order of $\zeta\left(\frac{1}{2}+i t\right)$, Ann. Scuola Norm. Sup. Pisa 13 (1986), 449-472.

[3] J.-R. Chen, On the representation of a large even integer as the sum of a prime and the product of at most two primes, Sci. Sinica 16 (1973), 157-176.

[4] H. Davenport, Multiplicative Number Theory (revised by H. Montgomery), 2nd ed., Springer, 1980.

[5] R. R. Hall and G. Tenenbaum, Divisors, Cambridge Univ. Press, 1988.

[6] D. R. Heath-Brown, Three primes and an almost-prime in arithmetic progression, J. London Math. Soc. (2) 23 (1981), 396-414.

[7] -, Prime numbers in short intervals and a generalized Vaughan identity, Canad. J. Math. 34 (1982), 1365-1377.

[8] L.-K. Hua, Some results in the additive prime number theory, Quart. J. Math. Oxford 9 (1938), 68-80.

[9] - Introduction to Number Theory, Springer, 1982.

[10] H. Iwaniec, On sums of two norms from cubic fields, in: Journées de théorie additive des nombres, Université de Bordeaux I, 1977, 71-89.

[11] —, Roser's sieve, Acta Arith. 36 (1980), 171-202.

[12] -, A new form of the error term in the linear sieve, ibid. 37 (1980), 307-320.

[13] M. B. S. Laporta and D. I. Tolev, On the sum of five squares of primes, one of which belongs to an arithmetic progression, unpublished.

[14] M.-C. Leung and M.-C. Liu, On generalized quadratic equations in three prime variables, Monatsh. Math. 115 (1993), 113-169.

[15] J. Liu and T. Zhan, On a theorem of Hua, Arch. Math. (Basel) 69 (1997), 375-390.

[16] H. Mikawa, On the sum of three squares of primes, in: Analytic Number Theory, London Math. Soc. Lecture Note Ser. 247, Cambridge Univ. Press, 1997.

[17] H. L. Montgomery, The analytic principle of the large sieve, Bull. Amer. Math. Soc. 84 (1978), 547-567.

[18] H. L. Montgomery and R. C. Vaughan, Hilbert's inequality, J. London Math. Soc. (2) 8 (1974), 73-82.

[19] - - - The exceptional set in Goldbach's problem, Acta Arith. 27 (1975), 353-370.

[20] T. P. Peneva, On the ternary Goldbach problem with primes $p_{i}$ such that $p_{i}+2$ are almost-primes, to appear.

[21] T. P. Peneva and D. I. Tolev, An additive problem with primes and almost-primes, Acta Arith. 83 (1998), 155-169.

[22] W. Schwarz, Zur Darstellung von Zahlen durch Summen von Primzahlpotenzen, II, J. Reine Angew. Math. 206 (1961), 78-112.

[23] D. I. Tolev, Arithmetic progressions of prime-almost-prime twins, Acta Arith. 88 (1999), 67-98.

[24] - On the representation of an integer as a sum of five squares of prime numbers of a special type, preprint, Plovdiv University, 2, March, 1998.

[25] - Representations of large integers as sums of two primes of special type, in: Algebraic Number Theory and Diophantine Analysis (Graz, 1998), de Gruyter, 2000, 485-495.

[26] J. G. van der Corput, Über Summen von Primzahlen und Primzahlquadraten, Math. Ann. 116 (1939), 1-50. 
[27] R. C. Vaughan, The Hardy-Littlewood Method, 2nd ed., Cambridge Univ. Press, 1997.

[28] I. M. Vinogradov, Representation of an odd number as a sum of three primes, Dokl. Akad. Nauk SSSR 15 (1937), 169-172 (in Russian).

Department of Mathematics

Plovdiv University "P. Hilendarski"

"Tsar Asen" 24

Plovdiv 4000, Bulgaria

E-mail: dtolev@ulcc.uni-plovdiv.bg

Received on 27.7.1999

and in revised form 20.1.2000

Added in proof (September 2000). After the present paper was submitted for publication Professor H. Mikawa sent to the author the manuscript On exponential sums over primes in arithmetic progressions. In this article he establishes non-trivial estimates for the sums

$$
\sum_{(d, c)=1} \lambda(d) \sum_{n \leq x, n \equiv c(d)} \Lambda(n) e(\alpha n)
$$

where $\alpha$ belongs to the set of minor arcs, $c \neq 0$ is a fixed integer, $\lambda$ is any well-factorable function of level $x^{4 / 9}(\log x)^{-B}$ and $B>0$. This result implies a slight improvement of Theorem 2 and Corollaries 2 and 3. The method can be used to improve also Theorem 1. However the calculations will be quite difficult.

The author would like to thank Professor H. Mikawa for informing about his result and sending the manuscript. 